\title{
LOCATING THE BAKING ISOTHERM IN A SØDERBERG ELECTRODE: ANALYSIS OF A MOVING THERMISTOR MODEL
}

\author{
ROBERT A. VAN GORDER*, ALISSA KAMILOVA ${ }^{\dagger}$, ROLF G. BIRKELAND ${ }^{\ddagger}$, AND ANDREW L. KRAUSE ${ }^{\dagger}$
}

Abstract. Søderberg electrodes feature prominently in the operation of metallurgical electrical furnaces. The electrode material must bake before entering the furnace; failure to bake will lower the efficiency of the process, and may cause physical harm to the furnace itself through a soft breakage. As such, ensuring that the baking isotherm remains within the region of the electrode outside of the furnace is essential. We propose a mathematical model for a Søderberg electrode taking into account the heat, mass, and current transfer mechanisms at play, along with realistic boundary conditions on the outside of the electrode that are strongly heterogeneous in height. The resulting model describes a strongly heterogeneous cylindrical "thermistor" which moves slowly downward and is acted on by current clamps which provide Joule heating. Although it is often ignored in the literature on thermistor problems, we find that the Péclet number resulting from the downward motion strongly influences the position of the baking isotherm. Aside from some specific reductions leading to analytical solutions, the general form of the model is complicated enough to require numerical simulations. Still, our modeling approach provides us with a qualitative understanding of many aspects of the Søderberg electrode baking process, and permits us to identify three parameters of key importance to the positioning of the baking isotherm. In particular, our results suggest desired ranges for the the lowering rate of the electrode (in terms of a Péclet number), the radius of the electrode, and the strength of the Joule heating due to an applied current, which are the three aspects which may be controlled (to varying degrees) in industrial applications.

Key word. Søderberg electrode; thermistor; baking isotherm; soft breakage

AMS subject classifications. 80A20, 76W05, 35Q79, 35Q60

1. Introduction. The Søderberg electrode was invented in 1919 by the company now known as Elkem [45] and is the most commonly used continuously consumed electrode system for providing the energy necessary for the production of ferroalloys and calcium carbide [3]. The electrodes conduct currents of up to $150 \mathrm{kA}$ to the centre of a smelting furnace, and operate at temperatures of up to $2000^{\circ} \mathrm{C}$ [23]. Although the design of Søderberg electrodes has not been significantly changed, product demand has led to an increase in the dimensions and current load [20]. The electrode is commonly enclosed in a thin steel casing which is lowered at specific times. For simplicity, an approximated continuous rate called the slipping rate is used to describe the speed at which the casing is lowered into the furnace, commonly at about $0.5 \mathrm{~m}$ per day $[3,35]$. A paste composed of a mixture of pitch tar and binder is the raw material that makes up the electrodes. This paste is viscoelastic [11, 47] and has been described as a shear thinning fluid [29]. The paste constituting an electrode can take the form of solid cylinders, briquettes, or blocks. Heat transfer in the electrode is due to the heat supplied by fans blowing hot air along the walls as well as heat induced by a current supplied by copper tubes to the current clamps (also called contact shoes) pressed against the outside of the casing. As the temperature increases into the range $400-500^{\circ} \mathrm{C}$, the paste softens, flows, and finally bakes [5]. The flow of the paste is primarily due to the addition of new electrode material at the top of the domain. The location within the electrode where the paste bakes is referred to as the baking isotherm. There are steel plates that surround the inside of the casing, referred to as fins. The softened paste fills the space between the fins and then becomes attached to the fins as it bakes, at which point the fins support the weight of the resulting baked electrode.

As the paste is baked, it becomes highly conductive and enables the electrode to conduct current to the tip where the arcs are formed. The arcs are the primary source of heat that powers the smelting process. Note that the electrode is consumed at the bottom as more material is added at the top. The correct melting and baking of paste is essential to produce electrodes with the desired mechanical, thermal, and electrical properties on which the entire production process depends [23, 29]. Various aspects of Søderberg electrode operation have been investigated, both numerically and analytically, over the years. The temperature distribution within Søderberg electrodes has been studied numerically by [21] and [37], matching observations made by Elkem [23]. More recently, in [35] a Comsol model was used to determine that the steel fins fitted in the casing are indispensable for its heating profile, as the heat conduction by the steel fins counteracts the

* Department of Mathematics and Statistics, University of Otago, P.O. Box 56, Dunedin 9054, New Zealand (rvangorder@maths.otago.ac.nz)

${ }^{\dagger}$ Mathematical Institute, University of Oxford, Andrew Wiles Building, Radcliffe Observatory Quarter, Woodstock Road, Oxford, OX2 6GG, United Kingdom

${ }^{\ddagger}$ Elkem ASA, Technology, Fiskaaveien 100, 4621 Kristiansand, Norway 


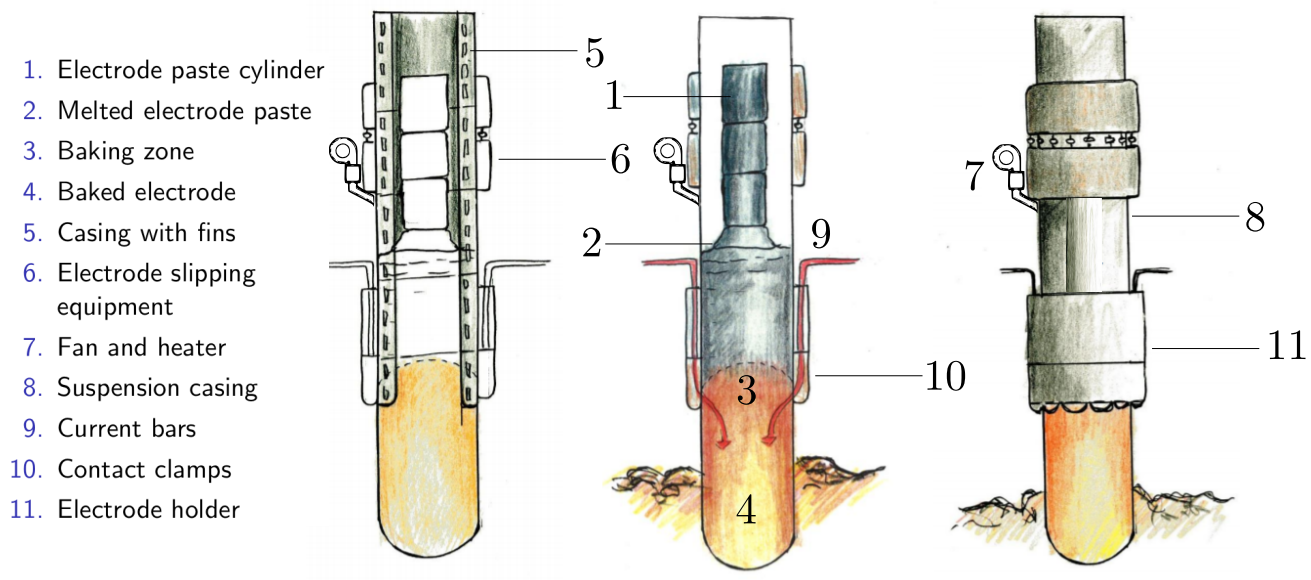

Fig. 1: Detailed schematic of a Søderberg electrode adapted from [15] to include labels and hot air fan.

induction heating and subsequent current clamp cooling mechanism. The fins also keep the baked electrode from falling into the furnace too fast, as the softened paste fills the space between the current fins and becomes attached to them during the baking process [42]. A detailed schematic of the electrode is shown in Figure 1.

Paste segregation can occur when the paste softening is out of balance. The causes of this phenomenon are not well understood, but it is known that the segregation problem began to occur more frequently when changes in design led to a decrease in the space between the copper tubes that supply the current to the clamps and the electrode casing, resulting in unwanted electrical induction within the cylinder [36]. In [3], billets of paste were modelled as a highly viscous two-phase particulate medium. The mechanism of segregation was taken to be the separation between the coarse and fine parts of the paste, and segregation was assumed to occur near the walls of the electrode casing. It was discovered that segregation occurs when the paste at the top of the electrode is so viscous that it does not deform from the incoming billets. Although segregation will not be directly investigated in this paper, it is an issue that leads to breakages in the electrodes, which are the failure mechanisms addressed in this work. In [12], a briquette model was derived taking a two-phase slow flow approach in order to understand how the paste briquettes soften while interacting with the air surrounding them. The results of [12] were compared to an experiment conducted by Elkem, and it was found that accounting for variations in viscosity is necessary for accurate predictions.

The electrical problem for the Søderberg electrode has been studied as a thermistor problem, which describes the heat produced in a conductor by an electric current. An upper bound on the temperature in such problems for simple boundary conditions was obtained in [9]. Additional posedness and blow-up results followed in [1]. Thermal runaway as a route to blow-up in the time-dependent form of these problems was further elucidated in $[25,26]$. A transient surge in temperature (similar to thermal runaway, yet selfsaturating) was shown in [13] to lead to cracking of thermistors (cracking is observed in real thermistors). A geometric reduction like that used in [10] was studied in two space dimensions in terms of a conformal map from the thermistor domain onto a rectangle [18].

There are two types of breakages that can occur in the operation of Søderberg electrodes, and we show diagrams of these in Fig. 2. A hard breakage occurs when large parts of baked carbon fall into the furnace. These are common during furnace shutdowns and are believed to be caused by an increase in thermal stresses which occur during the cooling and heating of an electrode. Stress levels increase with longer shutdown times, and have been investigated numerically by [30]. Some control strategies, e.g. shorter shutdowns, proper thermal insulation of the electrode surface, and allowing the electrode to slip after turning off the power, were suggested as a result of this study. Furthermore, a partially baked Søderberg electrode is especially susceptible to breakages caused by thermal shocks as the raw material of the paste will have different thermal dimensional behaviours which will impart thermal stress in the electrode [5]. A soft breakage occurs when the entire baked part of the electrode (and sometimes the whole electrode) plunges into the 

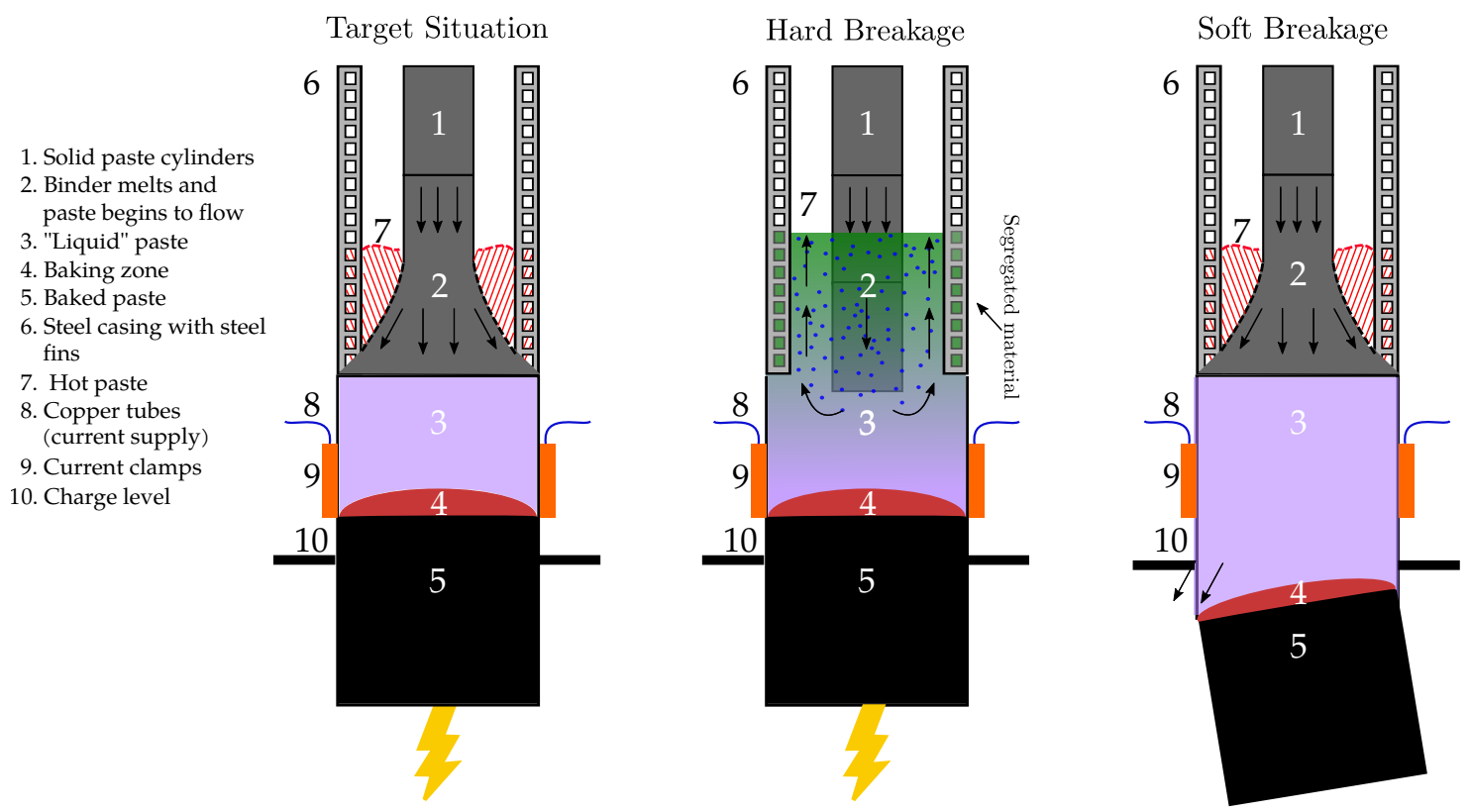

Fig. 2: Graphical representation of the problem to be considered. The target situation shows the normal operating configuration of the electrode provided that the baking isotherm closes appropriately. If the baking isotherm fails to close, which is the soft breakage case, paste may seep into the furnace, resulting in costly damage. A hard breakage is also possible, but we do not consider that case in the present work.

furnace. These are considered more serious than hard breakages and are costly to repair. Case studies have found that the main causes for soft breakages are a fast slipping rate, a low baking isotherm which fails to close above the top of the furnace region, and a high current in the steel casing [38].

In recent years, Elkem have dedicated resources to research projects aimed at improving their silicon production process. With regard to the Søderberg electrode, Elkem are interested in further improving their electrode operating strategies with a better understanding of the baking isotherm, in order to prevent the problems described above. Most of the aforementioned work on the baking isotherm present in the literature has involved CFD-type simulations, yet there has been relatively little mathematical modelling work done. While CFD-type simulations can be useful for understanding solutions corresponding to very specific physical conditions, mathematical modelling is often useful for determining the role that various physical parameters play in modifying or controlling the salient features of a solution. Elkem and the University of Oxford have collaborated on a variety of mathematical modelling projects in the past, and the particular project resulting in the work described herein originated in response to two Study Groups with Industry which took place in 2018. Work leading to the present paper started later in 2018, out of a desire to explore a different modelling approach to what was considered in the aforementioned Study Groups with Industry, with continuing discussions with Elkem and comparisons with data informing the modelling efforts. The mathematical modelling and analytical results obtained in the resulting collaboration point to a few key parameter groups which are useful in the control of the electrode baking process. These features are not always apparent from block-box or CFD simulations. The results are therefore of great practical importance to Elkem, and to anyone else interested in the operation of Søderberg electrodes.

Motivated by the application of baking a Søderberg electrode which is lowered into a submerged arc furnace, we develop a mathematical model for the heat, mass, and current transfer taking place within a Søderberg electrode, highlighting work arising out of the Elkem - University of Oxford collaboration discussed above. With this modelling work, we aim to bridge the gap between existing simple thermistor and electrode models and the more complicated yet realistic operating conditions of relevance to industrial applications (which are at present just approximated through CFD simulations), in order to identify the baking isotherm location and prevent costly soft breakages. The model is presented in Section 2. The model solutions are then 
studied analytically and through numerical simulations in Section 3. Despite the complexity of the model, we are able to both qualitatively and quantitatively determine the dependence of the baking isotherm on (i) the lowering rate of the electrode (through a Péclet number); (ii) the radius of the electrode; and (iii) the strength of the Joule heating due to an applied current, which are the three aspects that may be controlled in industrial applications. We discuss these qualitative findings in Section 4, highlighting those findings and recommendations relevant to furnace operators, and suggesting a variety of extensions of our model for sake of greater accuracy or other applications.

2. Mathematical model. We consider a model for the melting and the solidification of paste in a Søderberg electrode under the assumption of cylindrical geometry of fixed radius. Comparing this with the graphical representation in Figure 2, we consider a model valid for the left and right cases where the furnace is operating properly, and hence the paste cylinder does not sink into the pool of segregated hot paste, shown in green, meaning that the cylindrical geometry is a sensible approximation. We treat the entire domain as one fluid, with very high viscosity in the "solid" regions.

We start with the compressible Navier-Stokes, energy, and current equations

$$
\begin{gathered}
\frac{\partial \hat{\rho}}{\partial \hat{t}}+\nabla \cdot(\hat{\rho} \hat{\mathbf{u}})=0, \\
\hat{\rho} \frac{\partial \hat{\mathbf{u}}}{\partial \hat{t}}+\hat{\rho} \hat{\mathbf{u}} \cdot \nabla \hat{\mathbf{u}}=\nabla \cdot\left(\hat{\mu}\left(\nabla \hat{\mathbf{u}}+\nabla \hat{\mathbf{u}}^{\top}\right)\right)-\frac{2}{3} \nabla(\hat{\mu} \nabla \cdot \hat{\mathbf{u}})-\nabla \hat{p}-\hat{\rho} g \mathbf{e}_{\hat{z}}, \\
\hat{\rho} c_{p} \frac{\partial \hat{T}}{\partial \hat{t}}+\hat{\rho} c_{p} \hat{\mathbf{u}} \cdot \nabla \hat{T}=\nabla \cdot(\hat{k}(\hat{T}) \nabla \hat{T})+\hat{\sigma}(\hat{T})|\nabla \hat{\phi}|^{2}+\alpha \hat{T}\left(\frac{\partial \hat{p}}{\partial \hat{t}}+\hat{\mathbf{u}} \cdot \nabla \hat{p}\right) \\
+\left(\nabla \cdot\left(\hat{\mu}\left(\nabla \hat{\mathbf{u}}+\nabla \hat{\mathbf{u}}^{\top}\right)\right)-\frac{2}{3} \nabla(\hat{\mu} \nabla \cdot \hat{\mathbf{u}})\right): \nabla \hat{\mathbf{u}}, \\
\nabla \cdot(\hat{\sigma}(\hat{T}) \nabla \hat{\phi})=0 .
\end{gathered}
$$

Here $\hat{u}$ is the velocity field, $\hat{\rho}$ is the density, $\hat{\mu}$ is the viscosity, $\hat{p}$ is the pressure, $\hat{T}$ is the temperature, $\hat{\phi}$ is the electric potential, while $c_{p}$ is the heat capacity, $\alpha$ is the coefficient of thermal expansion, and $g$ is the acceleration due to gravity. The electrical conductivity, $\hat{\sigma}$ viscosity $\hat{\mu}$, thermal conductivity $\hat{k}$ are in general functions of temperature. The fluid system is subject to an equation of state $\mathcal{F}(\hat{p}, \hat{\rho}, \hat{T})=0$. For a more thorough treatment and derivation of these various terms in (2)-(3), see [2, 28]. We provide a list of reference parameter values in Table 1. There is Joule heating within the cylinder due to the current clamps, and this is responsible for the heat source proportional to the norm of the current density, $\nabla \hat{\phi}$, in (3). Although we consider a time-dependent problem for the heat and mass transfer, we assume that the electrical problem remains static. This is reasonable, as the time-dependent electrical problem equilibrates instantaneously relative to the other components of the problem.

We are most interested in using this model to better understand the placement of the baking isotherm within the electrode. If this isotherm fails to close before it reaches what we designate the bottom of the electrode, then costly soft breakages may result. The electrode paste begins to melt at between $50 \mathrm{C}$ and $100 \mathrm{C}$, while baking is completed at between $400 \mathrm{C}$ and $500 \mathrm{C}$ [5]. Thermomechanical analysis was used to measure the dimensional changes that take place in coal tar pitch in the temperature range relevant to the determination of the baking isotherm temperature by [41], suggesting that the isotherm for a fully baked material lies in the range $400 \mathrm{C}-450 \mathrm{C}$. The precise range will depend on the components of the paste. For instance, a combination of coal tar pitch, granular anthracite, and organic binder is used in some ramming paste, and this is usually baked by $400 \mathrm{C}$ [39]. For the material Elkem uses, softening of the paste has been known to occur near a temperature of $80 \mathrm{C}$, and baking is believed to begin to take place at $350 \mathrm{C}$, based on discussions with Elkem. At this temperature, even if not fully baked, the paste is solid enough to prevent flow of liquid paste into the furnace below. Furthermore, for the temperature values described above, we do not include radiation effects, as these are only significant near the bottom of the electrode where the electric arcs are formed and temperatures are much higher $(>2000 \mathrm{C})$.

2.1. Boundary conditions in cylindrical coordinates. We restrict our attention to a circular cylinder, and consider an axisymmetric cylindrical coordinate frame $(\hat{r}, \hat{z})$. The velocity field is then given by $\hat{\mathbf{u}}=(\hat{u}, \hat{v})$, where $\hat{u}$ is the velocity component in the $\mathbf{e}_{\hat{r}}$ direction and $\hat{v}$ is the velocity component in the 


\begin{tabular}{|c|l|c|c|}
\hline Notation & Parameter Name & Value & Reference \\
\hline$\hat{H}$ & Height of Electrode & $7 \mathrm{~m}$ & {$[42]$} \\
\hline$\hat{R}$ & Radius of Electrode Casing & $1 \mathrm{~m}$ & {$[3]$} \\
\hline$\hat{T}_{\text {cool }}$ & Air Temperature & $338 \mathrm{~K}$ & - \\
\hline$\hat{T}_{\text {melt }}$ & Melting Temperature & $\approx 353 \mathrm{~K}$ & - \\
\hline$\hat{T}_{\text {bake }}$ & Baking Temperature & $623 \mathrm{~K}$ & - \\
\hline$\hat{v}_{s}$ & Slipping Rate (Velocity Scale) & $5 \times 10^{-6}-3 \times 10^{-3} \mathrm{~m} \mathrm{~s}^{-1}$ & {$[3]$} \\
\hline$\hat{p}_{\text {ref }}$ & Boundary Pressure & $1 \mathrm{~atm}^{-1}$ & - \\
\hline $\bar{\mu}$ & Reference Viscosity Scale & $8.627 \times 10^{2} \mathrm{~kg} \mathrm{~s}^{-1} \mathrm{~m}^{-1}$ & - \\
\hline $\bar{\rho}$ & Reference Density Scale & $1.8 \times 10^{3} \mathrm{~kg} \mathrm{~m}^{-3}$ & {$[3]$} \\
\hline $\bar{k}$ & Reference Thermal Conductivity Scale & $5 \mathrm{~W} \mathrm{~m}^{-1} \mathrm{~K}^{-1}$ & {$[4,34]$} \\
\hline$c_{p}$ & Heat Capacity & $900 \mathrm{~J} \mathrm{~kg}^{-1} \mathrm{~K}^{-1}$ & {$[12]$} \\
\hline $\bar{h}$ & Coefficient of Convective Heat Transfer & $15 \mathrm{~W} \mathrm{~m}^{-2} \mathrm{~K}^{-1}$ & Elkem \\
\hline$I$ & Electric Current from Clamps & $80000 \mathrm{~A}^{-1}$ & {$[31]$} \\
\hline $\bar{\sigma}$ & Reference Electrical Conductivity & $32000 \Omega^{-1} \mathrm{~m}^{-1}$ & {$[27,31]$} \\
\hline$g$ & Gravity & $9.81 \mathrm{~m} \mathrm{~s}^{-2}$ & - \\
\hline$\alpha$ & Coefficient of Thermal Expansion & $\mathcal{O}\left(10^{-5}\right)-\mathcal{O}^{-1}\left(10^{-6}\right) \mathrm{K}^{-1}$ & {$[31]$} \\
\hline
\end{tabular}

Table 1: Reference values for dimensional parameters. Parameters without references were obtained through discussions with Elkem.

$\mathbf{e}_{\hat{z}}$ direction. Boundary conditions for the fluid problem are

$$
\begin{array}{r}
\frac{\partial \hat{v}}{\partial \hat{z}}=0, \quad \frac{\partial \hat{u}}{\partial \hat{z}}+\frac{\partial \hat{v}}{\partial \hat{r}}=0, \quad \hat{p}=\hat{p}_{\text {ref }} \text { at } \hat{z}=\hat{H}, \\
\hat{\mathbf{u}}=\left(0,-\hat{v}_{s}\right) \text { at } \hat{z}=0, \\
\hat{\mathbf{u}} \text { bounded at } \hat{r}=0, \\
\hat{\mathbf{u}}=\left(0,-\hat{v}_{s}\right) \text { at } \hat{r}=\hat{R} .
\end{array}
$$

The cylinder slips with velocity $\hat{v}_{s}$ as the electrode we model is lowered into the furnace. In real furnace operation, $\hat{v}_{s}$ is a function of time, with the electrode periodically stopped and then slipping with a velocity of near $\hat{v}_{s}=\mathcal{O}\left(10^{-3}\right) \mathrm{m} \mathrm{s}^{-1}$. In simulations, a slower averaged slip which is constant in time is employed, in which case $\hat{v}_{s}=\mathcal{O}\left(10^{-5}\right) \mathrm{m} \mathrm{s}^{-1}$.

Boundary conditions for the thermal problem read

$$
\begin{aligned}
& \hat{T}=\hat{T}_{\text {cool }} \quad \text { at } \quad \hat{z}=\hat{H}, \\
& \frac{\partial \hat{T}}{\partial \hat{z}}=0 \quad \text { at } \quad \hat{z}=0, \\
& \hat{T} \text { bounded at } \hat{r}=0 \text {, } \\
& \hat{k}(\hat{T}) \frac{\partial \hat{T}}{\partial \hat{r}}=\hat{h}(\hat{z})\left(\hat{T}_{\text {ext }}(\hat{z})-\hat{T}\right) \quad \text { at } \quad \hat{r}=\hat{R} .
\end{aligned}
$$

The electrode temperature is the cool ambient temperature at the top. The condition (6d) is just Newton's Law of Cooling at the radial boundary [14]. At the bottom, in practice the electrode reaches a steady temperature in equilibrium with the furnace, and we assume that there is no change in temperature at $\hat{z}=0$. This is not the same as no-flux, as there will in principle be heat transfer from the electrode into the furnace along with current transfer.

There are current clamps along the boundary of the cylinder (say, within the region $\hat{z} \in\left[\hat{H}_{1}, \hat{H}_{2}\right]$, $0 \leq \hat{H}_{1}<\hat{H}_{2} \leq \hat{H}$ at $\hat{r}=1$ ) providing a current $I$, and we assume that the bottom of the electrode is effectively grounded. This permits current to flow from the current clamps, through the electrode, and into 


\begin{tabular}{|c|c|c|c|}
\hline Notation & Parameter Group & Definition & Value \\
\hline$a$ & Aspect Ratio & $\frac{R}{H}$ & $0.15-0.25$ \\
\hline Stk & Stokes Number & $\frac{\bar{\rho} g \hat{R}^{2}}{\bar{\mu} \hat{v}_{s}}$ & $\mathcal{O}\left(10^{3}\right)-\mathcal{O}\left(10^{6}\right)$ \\
\hline $\operatorname{Re}$ & Reynolds Number & $\frac{\bar{\rho} \hat{\rho} \hat{v}_{s}}{\bar{\mu}}$ & $\mathcal{O}\left(10^{-6}\right)-\mathcal{O}\left(10^{-3}\right)$ \\
\hline $\mathrm{Pe}$ & Péclet Number & $\frac{R \hat{v}_{s}}{\bar{k} /\left(\bar{\rho} c_{p}\right)}$ & $\mathcal{O}\left(10^{-1}\right)-\mathcal{O}\left(10^{3}\right)$ \\
\hline $\mathrm{Nu}$ & Nusselt Number & $\frac{\bar{h}}{(\bar{k} / \hat{R})}$ & 5 \\
\hline Po & Pomerantsev Number & $\frac{I^{2}}{4 \pi^{2}\left(\hat{H}_{2}-\hat{H}_{1}\right)^{2} \bar{k} \bar{\sigma}\left(\hat{T}_{\text {bake }}-\hat{T}_{\text {cool }}\right)}$ & $\mathcal{O}(1)-\mathcal{O}(10)$ \\
\hline $\operatorname{Pr}$ & Prandtl Number & $\frac{c_{p} \bar{\mu}}{\bar{k}}$ & $\mathcal{O}\left(10^{5}\right)$ \\
\hline Ec & Eckert Number & $\frac{\hat{v}_{s}^{2}}{c_{p}\left(\hat{T}_{\text {bake }}-\hat{T}_{\text {cool }}\right)}$ & $\mathcal{O}\left(10^{-16}\right)-\mathcal{O}\left(10^{-11}\right)$ \\
\hline $\mathrm{Br}$ & Brinkman Number & $\frac{\bar{\mu} \hat{v}_{s}^{2}}{\overline{\bar{k}\left(\hat{T}_{\mathrm{bake}}-\hat{T}_{\text {cool }}\right)}}$ & $\mathcal{O}\left(10^{-11}\right)-\mathcal{O}\left(10^{-6}\right)$ \\
\hline$T_{\text {cool }}$ & Dimensionless Temperature at $z=0$ & - & 0 \\
\hline$T_{\text {melt }}$ & Dimensionless Melting Temperature & $\frac{T_{\text {melt }}-T_{\text {cool }}}{\hat{T}_{\text {bake }}-\hat{T}_{\text {cool }}}$ & $5.263 \times 10^{-2}$ \\
\hline$T_{\text {bake }}$ & Dimensionless Baking Temperature & - & 1 \\
\hline
\end{tabular}

Table 2: Reference values for useful dimensionless parameter groups and other dimensionless quantities.

the furnace below. A sensible set of boundary conditions for the electric potential then takes the form

$$
\hat{\sigma}(\hat{T}) \frac{\partial \hat{\phi}}{\partial \hat{r}}=\left\{\begin{array}{l}
\frac{I}{2 \pi \hat{R}\left(\hat{H}_{2}-\hat{H}_{1}\right)} \\
0
\end{array}\right.
$$

$$
\begin{array}{r}
\hat{\sigma}(\hat{T}) \frac{\partial \hat{\phi}}{\partial \hat{z}}=0 \quad \text { at } \quad \hat{z}=\hat{H}, \\
\hat{\phi}=0 \quad \text { at } \quad \hat{z}=0,
\end{array}
$$

For this problem, a current $I$ flows from the clamps, through the electrode, and out the bottom into the furnace. In (7c), the current is scaled over the area of the current clamp region, $2 \pi \hat{R}\left(\hat{H}_{2}-\hat{H}_{1}\right)$, to obtain a current density.

In practice, soft breakages may occur if the baking isotherm is very close to the bottom of the clamp, since there would not be enough mechanical strength to hold the material. We therefore note that, in practice, the critical height at which the baking isotherm must close may vary slightly. For our study, we take this critical height to be the bottom of the domain, and hence require that the baking isotherm close for some $\hat{z}>0$.

2.2. Scalings and dimensionless groups. Heat transfer mechanisms act at the boundary $\hat{r}=\hat{R}$ and heating effects penetrate inward. As such, it is sensible to nondimensionalise with respect to the radial length scale. We introduce the nondimensionalisation and scalings: $\hat{r}=\hat{R} r, \hat{z}=\hat{H} z, \hat{t}=\frac{\hat{R}}{\hat{v}_{s}} t, \hat{u}=\hat{v}_{s} u$, $\hat{v}=\hat{v}_{s} v, \hat{p}=\hat{p}_{\text {ref }}+\bar{\rho} g \hat{R} p, \hat{\rho}(\hat{T})=\bar{\rho} \rho(T), \hat{\mu}(\hat{T})=\bar{\mu} \mu(T), \hat{k}(\hat{T})=\bar{k} k(T), \hat{h}(\hat{z})=\bar{h} h(z), \hat{\sigma}(\hat{T})=\bar{\sigma} \sigma(T)$, $\hat{T}=\hat{T}_{\text {cool }}+\left(\hat{T}_{\text {bake }}-\hat{T}_{\text {cool }}\right) T, \hat{T}_{\text {ext }}=\hat{T}_{\text {cool }}+\left(\hat{T}_{\text {bake }}-\hat{T}_{\text {cool }}\right) T_{\text {ext }}, \hat{\phi}=\frac{I}{2 \pi \hat{R}\left(\hat{H}_{2}-\hat{H}_{1}\right) \bar{\sigma}} \phi$

These scalings result in multiple dimensionless groups. In Table 2, we list the various dimensionless groups along with tabulated reference values or ranges. The aspect ratio is defined by $a=\frac{\hat{R}}{\hat{H}}$. Data and simulations from Elkem suggest $a=O(1)$ or $a=O\left(10^{-1}\right)$ for realistic electrode geometries. (A radius of $1 \mathrm{~m}$ and height of $7 \mathrm{~m}$ appear to be most commonly used.)

For $\hat{v}_{s}$ of order $\mathcal{O}\left(10^{-6}\right)$ to $\mathcal{O}\left(10^{-3}\right)$, we have that Re ranges from $\mathcal{O}\left(10^{-6}\right)$ to $\mathcal{O}\left(10^{-3}\right)$. In the case where there is a constant lowering of the cylinder, which corresponds to $\hat{v}_{s}=\mathcal{O}\left(10^{-5}\right)$, the Reynolds number is at most $\mathcal{O}\left(10^{-5}\right)$, and can be neglected. On the other hand, if the cylinder is quickly lowered, and then 
held still for a much longer duration (as is done in practice), then $\hat{v}_{s}=\mathcal{O}\left(10^{-3}\right)$, and hence the Reynolds number may be as large as $\mathcal{O}\left(10^{-3}\right)$, in which case there may be some inertial effects. This suggests that we should ignore inertial terms for the case where the cylinder is uniformly lowered at a small velocity, yet may wish to retain inertial terms when considering a cylinder which is held in place, then more quickly lowered, before being held in place again.

The disparity noted in the range of $\mathrm{Pe}$ is also due to the wide range of velocities possible. In the case of a periodically lowered electrode, the instantaneous Péclet number can be as large as $\mathcal{O}\left(10^{2}\right)$ or $\mathcal{O}\left(10^{3}\right)$, yet this is only for a short duration, after which point the electrode is held fixed in place. As we shall discuss later, this corresponds to an effective Péclet number multiple orders of magnitude smaller. If the process is instead modelled by a uniform lowering of the electrode over time, then the Péclet number is of size $\mathcal{O}\left(10^{-1}\right)$ or $\mathcal{O}(1)$.

We have scaled the current like $\hat{\phi}=\frac{I}{2 \pi \hat{R}\left(\hat{H}_{2}-\hat{H}_{1}\right) \bar{\sigma}} \phi$. The Joule heating parameter is then given in terms of the Pomerantsev number, Po. Standard values of the current $I$ appear to range between $60000 \mathrm{~A}$ and $150000 \mathrm{~A}[22,31]$. The current is applied via current clamps around the entire radius of the cylinder, between heights $H_{1}=\hat{H}_{1} / \hat{H}$ and $H_{2}=\hat{H}_{2} / \hat{H}$. When carrying out numerical simulations, we non-dimensonalise the electrical problem recalling that $\hat{H}_{1}=1.5 \mathrm{~m}, \hat{H}_{2}=2.5 \mathrm{~m}$ for the configuration Elkem uses, while $\hat{H}=7 \mathrm{~m}$, so the non-dimensional range over which the current clamps are applied is $z \in\left[H_{1}, H_{2}\right]=[0.214,0.357]$.

From available literature, the linear coefficient of thermal expansion, $\alpha$, for the paste should be of the order $\mathcal{O}\left(10^{-5}\right)[23,34]$, hence the parameter group $\frac{\alpha \bar{\rho} \hat{v}_{s}}{\hat{R}}$ is of order $\mathcal{O}\left(10^{-7}\right)$ to $\mathcal{O}\left(10^{-4}\right)$ for $\hat{v}_{s}$ of order $\mathcal{O}\left(10^{-6}\right)$ to $\mathcal{O}\left(10^{-3}\right)$. Hence, this is a very small parameter group in all scenarios, and will be neglected.

The viscous dissipation parameter may be written as the product of Prandtl (Pr) and Eckert (Ec) numbers, which is denoted by the Brinkman number, Br, and we shall follow this notation. Note that $\mathrm{Br}=\mathcal{O}\left(10^{-6}\right)$ at most, so it may be tempting to neglect the Brinkman number since we have neglected the thermal expansion. However, while the thermal expansion parameter is isolated, the Brinkman number multiplies the viscosity. We have selected the viscosity scale to be such that $\bar{\mu}=\mathcal{O}\left(10^{3}\right)$, making the minimal value of $\mu(T)$ an $\mathcal{O}(1)$ quantity. However, the maximal order of $\mu(T)$ will reach values of $\mathcal{O}\left(10^{7}\right)$. Hence, the product $\operatorname{Br} \mu(T)$ has a possible maximal value which is $\mathcal{O}(10)$ in the case where the slip is of maximal order, $\hat{v}_{s}=\mathcal{O}\left(10^{-3}\right)$.

Under our nondimensionalisation, $\hat{T}_{\text {cool }}$ is taken to zero $\left(T_{\text {cool }}=0\right), \hat{T}_{\text {bake }}$ is taken to one $\left(T_{\text {bake }}=1\right)$, and $\hat{T}_{\text {melt }}$ is taken to $T_{\text {melt }}=0.05263$. We shall be interested in the behavior of the isotherms corresponding to the melting and baking temperatures, and in particular, we study how the curves representing these isotherms change position and structure as various model parameters are changed.

2.3. Reduction of the flow problem. Using the aforementioned parameter ranges as well as the boundary conditions on the flow, the flow problem simplifies greatly. In order to account for the equation of state $\mathcal{F}(\hat{p}, \hat{\rho}, \hat{T})=0$, we remark that discussions with Elkem suggest that the density $\rho(T)$ changes by at most $10 \%$ over the full range of temperatures, and we therefore consider the flow to be incompressible. Our equation of state then reduces to setting $\rho(T)$ equal to a constant. We set $\rho(T)=1$, as $\bar{\rho}$ holds the constant value of the dimensional $\hat{\rho}(\hat{T})$.

The boundary velocity parameter $\bar{v}_{s}$ should be viewed as an average slip rate. In practice, the electrode is held fixed for a time, and then moved downward over a short interval of time, after which it is then held fixed again. This process continues periodically. In order to account for the aforementioned time-dependence of the velocity boundary conditions, we should include inertial terms in the fluid model, which leads us to consider the incompressible Navier-Stokes system

$$
\begin{aligned}
\nabla \cdot \mathbf{u}=0, & \operatorname{Re}\left(\frac{\partial \mathbf{u}}{\partial t}+\mathbf{u} \cdot \nabla \mathbf{u}\right)=\nabla \cdot\left(\mu\left(\nabla \mathbf{u}+\nabla \mathbf{u}^{\top}\right)\right)-\operatorname{Stk}\left(\nabla p+\mathbf{e}_{z}\right), \\
& \frac{\partial v}{\partial z}=0, \quad a \frac{\partial u}{\partial z}+\frac{\partial v}{\partial r}=0, \quad p=0 \quad \text { at } \quad z=1 \\
& (u, v)=(0,-f(t)) \text { at } z=0, \\
& (u, v) \text { bounded at } r=0 \\
& (u, v)=(0,-f(t)) \text { at } r=1 .
\end{aligned}
$$

Here $f(t)$ is the function which approximates the motion of the casing over time. When the casing is 
stationary, we have $f(t)=0$, while when the casing is moving at its maximal velocity $\hat{v}_{s}$, we have $f(t)=1$.

The boundary conditions (8b)-(8e) imply a spatially uniform velocity field, and an exact solution to the fluid problem is then given by

$$
u=0, \quad v=-f(t), \quad p=\left(1-\frac{\operatorname{Re}}{\operatorname{Stk}} \dot{f}(t)\right) \frac{1-z}{a},
$$

where $\dot{f}$ denotes the time derivative of $f$. Since $\operatorname{Re}=\mathcal{O}\left(10^{-3}\right)$ at most, while Stk $=\mathcal{O}\left(10^{3}\right)$ at a minimum, we therefore have that $\frac{\mathrm{Re}}{\mathrm{Stk}}=\mathcal{O}\left(10^{-6}\right)$ at most. As such, the inertial term $\frac{\mathrm{Re}}{\mathrm{Stk}}$ is negligible (even for rapid variations in velocity when slipping motion starts or stops), and the same results would have been obtained from a quasi-static approximation from the Stokes equations.

It is common practice to consider a continuously lowered electrode, which is lowered at some averaged rate much lower than the periodic slip rate. In this case, $f(t)=1$ for all time, and in this regime $\hat{v}_{s}$ is small and viewed as the time average of a periodic slip velocity; in practice $\hat{v}_{s}=\mathcal{O}\left(10^{-5}\right)$ or smaller for this regime. In this limit, we have $\operatorname{Re}=\mathcal{O}\left(10^{-4}\right)$, so we set $\operatorname{Re}=0$ and neglect the inertial terms, with (8a) reducing to the Stokes equations. The boundary conditions are the same as those given in (8b)-(8e), with $f(t)=1$, and we obtain the simpler exact solution

$$
u=0, \quad v=-1, \quad p=\frac{1-z}{a} .
$$

Furthermore, for $\mu(T) \geq \mu_{0}>0$, a solution to the Stokes equation is unique [7], and hence the exact solution (10) is the unique solution. This is useful to note, so that we need not worry about a second steady solution.

Note that the viscous dissipation term in equation (3) is identically zero for the velocities specified in (9) or (10), and we henceforth neglect it, regardless of the particular value of Br. That is to say, we do not need to use the fact that $\mathrm{Br}$ is small to neglect viscous dissipation; rather, the viscous dissipation term is identically zero due to the spatial uniformity of the exact flow solution.

2.4. Constitutive relations and boundary conditions. The electrical conductivity $\hat{\sigma}(\hat{T})$ was taken as $\hat{\sigma}(\hat{T})=\bar{\sigma}(1+\exp ((1048 \mathrm{C}-\hat{T}) / 338 \mathrm{C}))^{-1}$ in [27] with reference value is $\bar{\sigma}=32000 \Omega^{-1} \mathrm{~m}^{-1}$, corresponding to the value of electrical conductivity at the temperature $2273 \mathrm{~K}[27,31]$. Putting the relation into non-dimensional form, we find $\hat{\sigma}(\hat{T})=\bar{\sigma} \sigma(T)$, with

$$
\sigma(T)=\frac{1}{1+\exp (10.923-4.385 T)} .
$$

Similarly, using reference values given in $[4,34]$ for the thermal conductivity, we choose $\hat{k}(\hat{T})=\bar{k} k(T)$ with a reference value $\bar{k}=5 \mathrm{Wm}^{-1} \mathrm{~K}^{-1}$ and sigmoid form

$$
k(T)=\frac{1+\exp (1.997)}{1+\exp (2.936-0.939 T)} .
$$

Based on operating conditions observed by Elkem at the external boundaries of the domain, we consider an external thermal profile of the form $\hat{T}_{\text {ext }}(\hat{z})=1173 \mathrm{~K}$ for $\hat{z} \in[0 \mathrm{~m}, 1.5 \mathrm{~m})$, which models the hot region near the bottom of the electrode. The region around the current clamps is cooled, and we have $\hat{T}_{\text {ext }}(\hat{z})=333 \mathrm{~K}$ for $\hat{z} \in[1.5 \mathrm{~m}, 2.5 \mathrm{~m})$, whereas the region above the current clamps is warmer, $\hat{T}_{\text {ext }}(\hat{z})=473 \mathrm{~K}$ for $\hat{z} \in[2.5 \mathrm{~m}, 3.5 \mathrm{~m})$. Above this, there is a cooling with height, and we have $\hat{T}_{\text {ext }}(\hat{z})=753 \mathrm{~K}-80 \frac{\mathrm{K}}{\mathrm{m}} \hat{z}$ for $\hat{z} \in[3.5 \mathrm{~m}, 5 \mathrm{~m})$ and finally $\hat{T}_{\text {ext }}(\hat{z})=353 \mathrm{~K}$ for the topmost region where $\hat{z} \in[5 \mathrm{~m}, 7 \mathrm{~m}]$. In dimensionless form, we then have

$$
T_{\text {ext }}(z)= \begin{cases}2.930 & \text { for } z \in[0,0.214), \\ -1.754 \times 10^{-2} & \text { for } z \in[0.214,0.357), \\ 4.737 \times 10^{-1} & \text { for } z \in[0.357,0.5), \\ 1.458-1.968 z & \text { for } z \in[0.5,0.714), \\ 5.263 \times 10^{-2} & \text { for } z \in[0.714,1] .\end{cases}
$$

The spatially varying coefficient of convective heat transfer is estimated from Elkem data, and takes the form $\hat{h}(\hat{z})=7 \mathrm{~W} \mathrm{~m}^{-2} \mathrm{~K}^{-1}$ for $\hat{z} \in[0 \mathrm{~m}, 1.5 \mathrm{~m})$, increases strongly in the current clamp region to $\hat{h}(\hat{z})=$ 
$75 \mathrm{~W} \mathrm{~m}^{-2} \mathrm{~K}^{-1}$ for $\hat{z} \in[1.5 \mathrm{~m}, 2.5 \mathrm{~m})$, decreases to $\hat{h}(\hat{z})=15 \mathrm{~W} \mathrm{~m}^{-2} \mathrm{~K}^{-1}$ for $\hat{z} \in[2.5 \mathrm{~m}, 5 \mathrm{~m})$, and finally decreases to zero above that. The dimensionless coefficient of convective heat transfer reads

$$
h(z)= \begin{cases}\frac{7}{15} & \text { for } z \in[0,0.214) \\ 5 & \text { for } z \in[0.214,0.357) \\ 1 & \text { for } z \in[0.357,0.714) \\ 0 & \text { for } z \in[0.714,1]\end{cases}
$$

We have taken the dimensional scale factor to be $\bar{h}=15 \mathrm{~W} \mathrm{~m}^{-2} \mathrm{~K}^{-1}$, which we use as the reference value for the convective heat transfer in the Nusselt number.

2.5. Moving thermistor model in cylindrical coordinates. Our domain is that of a circular cylinder of height one and radius one. We take an axisymmetric cylindrical coordinate frame $(r, z)$. We have

$$
\begin{aligned}
\operatorname{Pe}\left(\frac{\partial T}{\partial t}-a f(t) \frac{\partial T}{\partial z}\right)= & \frac{1}{r} \frac{\partial}{\partial r}\left(r k(T) \frac{\partial T}{\partial r}\right)+a^{2} \frac{\partial}{\partial z}\left(k(T) \frac{\partial T}{\partial z}\right)+\operatorname{Po} \sigma(T)\left(\left(\frac{\partial \phi}{\partial r}\right)^{2}+a^{2}\left(\frac{\partial \phi}{\partial z}\right)^{2}\right) \\
& \frac{1}{r} \frac{\partial}{\partial r}\left(r \sigma(T) \frac{\partial \phi}{\partial r}\right)+a^{2} \frac{\partial}{\partial z}\left(\sigma(T) \frac{\partial \phi}{\partial z}\right)=0
\end{aligned}
$$

Dimensionless boundary conditions for the thermal problem are

$$
\begin{aligned}
& T=0 \quad \text { at } \quad z=1, \\
& \frac{\partial T}{\partial z}=0 \quad \text { at } \quad z=0, \\
& T \text { bounded at } r=0 \text {, } \\
& k(T) \frac{\partial T}{\partial r}=\mathrm{Nu} h(z)\left(T_{\mathrm{ext}}(z)-T\right) \quad \text { at } \quad r=1 \text {, }
\end{aligned}
$$

and the electrical problem,

$$
\begin{aligned}
& \sigma(T) \frac{\partial \phi}{\partial z}=0 \quad \text { at } \quad z=1 \\
& \phi=0 \quad \text { at } z=0, \\
& \sigma(T) \frac{\partial \phi}{\partial r}=\left\{\begin{array}{ll}
1 & \text { for } z \in\left[H_{1}, H_{2}\right], \\
0 & \text { otherwise, }
\end{array} \quad \text { at } \quad r=1,\right. \\
& \phi \text { bounded at } r=0 \text {. }
\end{aligned}
$$

In the case where the slip is uniform, we take $f(t)=1$ and assume a steady state problem, replacing (15a) with

$$
-a \operatorname{Pe} \frac{\partial T}{\partial z}=\frac{1}{r} \frac{\partial}{\partial r}\left(r k(T) \frac{\partial T}{\partial r}\right)+a^{2} \frac{\partial}{\partial z}\left(k(T) \frac{\partial T}{\partial z}\right)+\operatorname{Po} \sigma(T)\left(\left(\frac{\partial \phi}{\partial r}\right)^{2}+a^{2}\left(\frac{\partial \phi}{\partial z}\right)^{2}\right) .
$$

We will refer to (18), (15a), (16), (17) as the stationary or steady form of the model. Note that although the temperature problem (15) is in general time-dependent, the electrical problem is kept static for all cases, as we assume that the electrical system equilibrates instantaneously relative to the thermal system even on the timescale of the electrode moving. As we are most interested in static or quasi-static dynamics, we do not fix the initial data, but comment that in simulations we explored a variety of initial data, all of which appeared to be within the basin of attraction for the steady (constant $f$ ) or quasi-steady (periodic $f(t)$ ) solutions.

If we consider the case of a static electrode which is not lowered, $\mathrm{Pe}=0$, we obtain the steady-state "thermistor" problem for a finite cylinder. Thermistor problems are well-studied in the literature, and we mention several relevant studies. An upper bound on the temperature in such problems (although with 
simpler boundary conditions than we employ) was obtained in [9]. For specific boundary conditions, systems of the form (18) and (15b) have been shown to admit either a unique solution or no solution, depending on the functions $k(T)$ and $\sigma(T)$ [10]. Additional posedness and blow-up results followed in [1]. Thermal runaway as a route to blow-up in the time-dependent form of these problems was further elucidated in $[25,26]$. A transient surge in temperature (similar to thermal runaway, yet self-saturating) may lead to cracking of thermistors (cracking is observed in real thermistors) [13]. A geometric reduction like that used in [10] was studied in two space dimensions in terms of a conformal map from the thermistor domain onto a rectangle [18]. Stationary solutions of (18) were further explored in [19] for certain boundary conditions. The degenerate case where $\sigma(T)=0$ for some $T$ was studied by [8]. While mathematically interesting and useful for motivating some of our findings, none of these studies considered complicated thermal boundary conditions such as (16). Furthermore, the role of thermal advection through a moving thermistor is ignored in much of the thermistor literature, yet this will be essential to consider for our study of a lowered electrode.

It has been remarked that convection will not matter in a qualitative sense for thermistor problems [27]. Although this is true for a more abstract mathematical formulation where length scales and boundaries do not matter, in practical applications the specific rate of lowering of an electrode modelled as a thermistor will play a strong role in the rate of heating and hence the position of the baking isotherm. In particular, fast rates of lowering may result in soft breakages, while excessively slow rates of lowering will be inefficient. As such, there is an interest in determining an optimal lowering rate, where an electrode is fed into a furnace at a rate which both prevents soft breakages yet preserves efficiency.

3. Qualitative and quantitative analysis of the model. In this section, we study the behavior of solutions to the model (15), (16), (17) both analytically and through numerical simulations. Our primary focus is on locating the baking isotherm, as soft breakages will occur if the baking isotherm goes beneath the bottom of the clamp. Regarding the simulations, we simulate the stationary form of these equations using the Finite Element solver COMSOL, using a mesh with 111, 202 second-order triangular mesh elements to approximate the domain. A non-uniform mesh is employed with a maximal element size of $3 \times 10^{-3}$ in a region of width 0.1 around the clamp, and a maximal element size of $5 \times 10^{-3}$ elsewhere. Convergence in the spatial discretization is checked by refining the mesh and computing norms of solution differences for select parameters. In order to determine how transient dynamics evolve into a steady state configuration, time-dependent simulations are computed using the same discretization as used in the stationary solver, and a relative error tolerance of $10^{-2}$, and the default adaptive time-stepping scheme using a generalized backward-difference formula of orders 1-5. All of the stationary solutions shown appear to be numerically stable when perturbed slightly and simulated forward in time. We find no evidence of multistability in the experimentally relevant parameter ranges.

In all simulations we fix $\mathrm{Nu}=5$, while varying the parameters $\mathrm{Pe}, a$, and Po within ranges of relevance to real furnace operation. In each case we compute the melting $\left(T=T_{\text {melt }}\right)$ and baking $(T=1)$ isotherms, and display these where they appear within the dimensionless geometry. In Figure 3 we plot a sample temperature profile, $T$, as well as the orders of magnitude of the distribution of the Joule heating, $\ln \left(1+|\nabla \phi|^{2}\right)$. In the example shown, the baking isotherm closes, and hence the electrode has successfully baked. Note that the observed drop-off in the electrode current density distribution as it penetrates the interior of the electrode is akin to observations from more complicated multiphysics simulations and experiments [22].

We first consider exact solutions for two strong simplifications of the model, in order to gain a qualitative understanding of the role played by the boundary conditions and Joule heating. After this, we consider the case of periodic slips, where the electrode is periodically lowered in time. We find that the dynamics over many such slips are akin to those from a stationary model (for reasonable duration slips), and explore the stationary case in more detail, providing parameter sweeps in the relevant dimensionless groups.

3.1. Exact solutions for two simplifications of the steady problem. We first consider simplification of the model (18), (15a), (16), (17), in order to gain better intuition for, and a qualitative understanding of, the steady problem.

3.1.1. Heat transfer in a lowered electrode in the absence of Joule heating. In order to better understand the role played by the combination of convective heating at the boundaries and thermal advection due to the slow downward motion of the electrode, we consider the limit $\mathrm{Po} \rightarrow 0$. To simplify our analysis, we take $k(T)=1$ and $h(z)=1$, since their reference values are already scaled out in the non-dimensionalization 


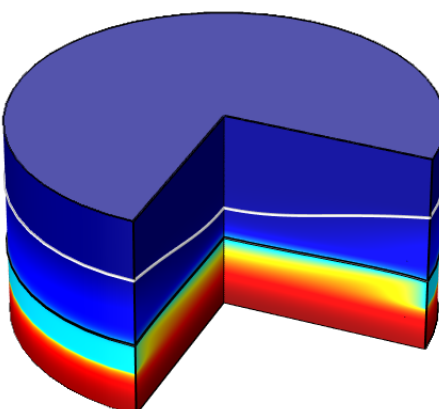

(a) $T$ (cylindrical geometry)

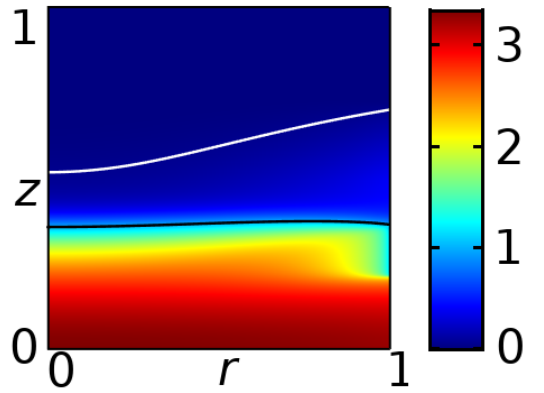

(b) $T$

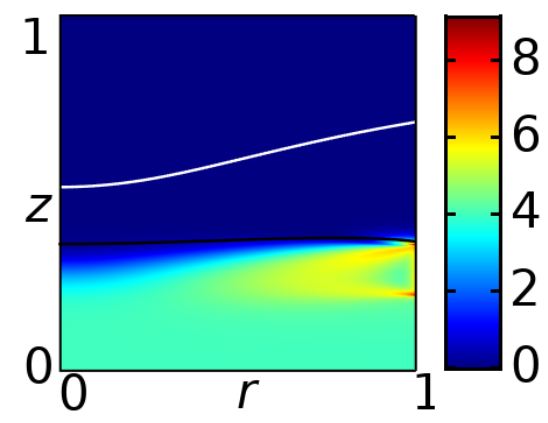

(c) $\ln \left(1+\sigma(T)|\nabla \phi|^{2}\right)$

Fig. 3: Numerical simulation of the system (18), (15a), (16), (17). (a) A 3D view of the temperature profile over a $270^{\circ}$-arc of the cylinder. (b) Heat map for the temperature in the $r-z$ coordinate frame. (c) Heat map for the logarithm of the Joule heating distribution given by $\ln \left(1+\sigma(T)|\nabla \phi|^{2}\right)$. All panels show isothermal surfaces corresponding to melting (white) and baking (black) isotherms. Parameter values are fixed at $a=0.14, \mathrm{Pe}=3, \mathrm{Po}=3.58$. This value of Po corresponds to an applied current of $80 \mathrm{kA}$.

presented in Section 2.2. We obtain from (18) the simplified problem

$$
-a \operatorname{Pe} \frac{\partial T}{\partial z}=\frac{1}{r} \frac{\partial}{\partial r}\left(r \frac{\partial T}{\partial r}\right)+a^{2} \frac{\partial^{2} T}{\partial z^{2}}, \quad \text { with } \quad \frac{\partial T}{\partial r}=\mathrm{Nu}\left(T_{\mathrm{ext}}(z)-T\right) \quad \text { at } \quad r=1,
$$

subject still to the boundary conditions (16a)-(16c). We transform the dependent variable, $T=\exp \left(-\frac{\mathrm{Pe}}{2 a} z\right) \theta$, which results in the modified problem for $\theta$ :

$$
\begin{gathered}
\frac{1}{r} \frac{\partial}{\partial r}\left(r \frac{\partial \theta}{\partial r}\right)+a^{2} \frac{\partial^{2} \theta}{\partial z^{2}}-\frac{\mathrm{Pe}^{2}}{4} \theta=0 \\
\frac{\partial \theta}{\partial z}-\frac{\mathrm{Pe}}{2 a} \theta=0 \text { at } z=0, \quad \theta=0 \text { at } z=1, \\
\theta \text { bounded at } r=0, \quad \frac{\partial \theta}{\partial r}=\mathrm{Nu}\left(\exp \left(\frac{\mathrm{Pe}}{2 a} z\right) T_{\operatorname{ext}}(z)-\theta\right) \text { at } r=1 .
\end{gathered}
$$

In order to solve (20), we first separate variables like $\theta(r, z)=\mathcal{R}(r) \mathcal{Z}(z)$, which gives the spectral problem

$$
a^{2} \frac{d^{2} \mathcal{Z}}{d z^{2}}-\frac{\mathrm{Pe}^{2}}{4} \mathcal{Z}=-\lambda^{2} \mathcal{Z}, \quad \frac{d \mathcal{Z}}{d z}-\frac{\mathrm{Pe}}{2 a} \mathcal{Z}=0 \quad \text { at } \quad z=0, \quad \mathcal{Z}=0 \quad \text { at } \quad z=1
$$

$$
\frac{1}{r} \frac{d}{d r}\left(r \frac{d \mathcal{R}}{d r}\right)=\lambda^{2} \mathcal{R}, \quad \mathcal{R} \text { bounded at } r=0,
$$

for $\lambda>0$. Note that the sign of the spectral term is the only possibility; replacing $-\lambda^{2}$ with $\lambda^{2}$ or $\lambda=0$ yields only the trivial solution $\mathcal{Z}(z) \equiv 0$. Solving (21a), we find

$$
\mathcal{Z}_{\ell}(z)=\cos \left(\Lambda_{\ell} z\right)+\frac{\mathrm{Pe}}{2 a \Lambda_{\ell}} \sin \left(\Lambda_{\ell} z\right), \quad \lambda_{\ell}=a \sqrt{\Lambda_{\ell}^{2}+\left(\frac{\mathrm{Pe}}{2 a}\right)^{2}},
$$

where the constants $\Lambda_{\ell}, \ell \geq 1$, are the positive roots of $\Lambda \cos (\Lambda)+\frac{P e}{2 a} \sin (\Lambda)=0$, arranged in ascending order. Rearranging, we have $\frac{2 a}{\mathrm{Pe}} \Lambda=-\tan (\Lambda)$. Since $-\tan (\Lambda) \rightarrow \infty$ as $\Lambda \rightarrow\left[\frac{(2 \ell-1) \pi}{2}\right]^{+}$and $-\tan (\ell \pi)=0$, each root $\Lambda_{\ell}$ must satisfy $\frac{(2 \ell-1) \pi}{2}<\Lambda_{\ell}<\ell \pi$. While there exist negative roots, these take the form $\Lambda_{-\ell}=-\Lambda_{\ell}$, hence $\mathcal{Z}_{-\ell}(z)=\mathcal{Z}_{\ell}(z)$, and by this symmetry it is sufficient to consider only $\ell \geq 1$. While there is a root $\Lambda_{0}=0$, this results in only the trivial solution $\mathcal{Z}_{0}(z) \equiv 0$. 


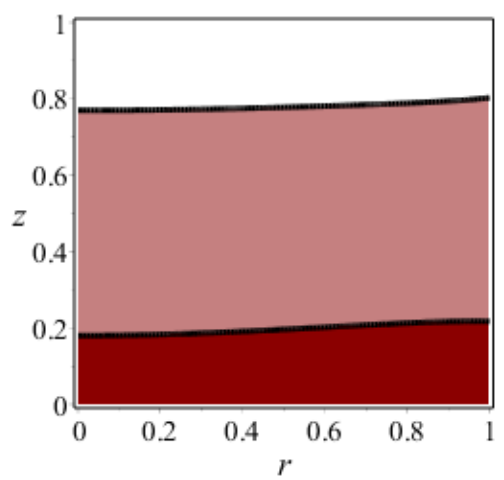

(a)

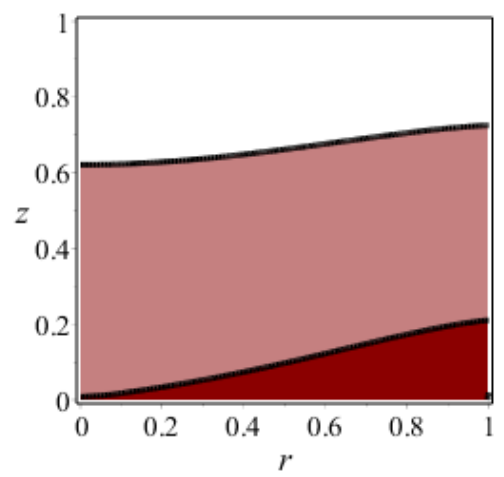

(b)

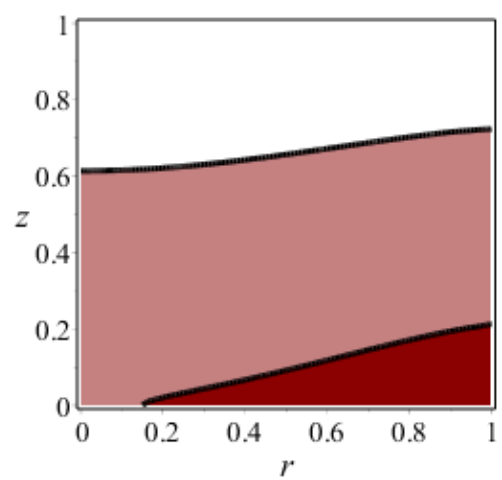

(c)

Fig. 4: Plots of the solution (23) for fixed aspect ratio $a=0.2$ and Nusselt number $\mathrm{Nu}=5$, given the Péclet numbers (a) $\mathrm{Pe}=2$, (b) $\mathrm{Pe}=5.3$, (c) $\mathrm{Pe}=5.5$. We plot the level curves corresponding to $T_{\text {melt }}=0.05$ and $T_{\text {bake }}=1$, with white regions corresponding to the solid region $T<T_{\text {melt }}$, light red regions corresponding to the melted region $T_{\text {melt }}<T<T_{\text {bake }}$, and dark red regions corresponding to the baked region $T_{\text {bake }}<T$.

We find from (21b) that $\mathcal{R}(r)=I_{0}\left(\lambda_{\ell} r\right)$, where $I_{0}$ denotes the relevant modified Bessel function of the first kind, is the solution which satisfies the ODE in the radial coordinate which is bounded for $r=0$. To complete the solution procedure, we write the Fourier series and place it into the remaining convective boundary condition. Evaluating this at $r=1$, we determine the remaining unknown coefficients by taking the Fourier expansion of $T_{\text {ext }}(z)$ in the basis functions $\mathcal{Z}_{\ell}(z)$ and matching the coefficients. Returning to $T$ from $\theta$, we obtain the exact solution in terms of the generalized Fourier series

$$
T(r, z)=\sum_{\ell=1}^{\infty} \mathcal{A}_{\ell} I_{0}\left(\lambda_{\ell} r\right)\left\{\cos \left(\Lambda_{\ell} z\right)+\frac{\mathrm{Pe}}{2 a \Lambda_{\ell}} \sin \left(\Lambda_{\ell} z\right)\right\} \exp \left(-\frac{\mathrm{Pe}}{2 a} z\right),
$$

where

$$
\mathcal{A}_{\ell}=\frac{\int_{0}^{1} \exp \left(\frac{\mathrm{Pe}}{2 a} \xi\right) T_{\operatorname{ext}}(\xi)\left\{\cos \left(\Lambda_{\ell} \xi\right)+\frac{\mathrm{Pe}}{2 a \Lambda_{\ell}} \sin \left(\Lambda_{\ell} \xi\right)\right\} d \xi}{\left(I_{0}\left(\lambda_{\ell}\right)+\frac{\lambda_{\ell}}{\mathrm{Nu}} I_{1}\left(\lambda_{\ell}\right)\right) \int_{0}^{1}\left[\cos \left(\Lambda_{\ell} \xi\right)+\frac{\mathrm{Pe}}{2 a \Lambda_{\ell}} \sin \left(\Lambda_{\ell} \xi\right)\right]^{2} d \xi} .
$$

In order to better understand the role of the Péclet number and aspect ratio in positioning the baking isotherm, we plot the series solution (23) in Figures 4-5. To do so, in Figure 4 we truncate the series at 300 terms, having verified that there is negligible difference between the 300 term and 1000 term truncation for these cases. More accuracy is needed to obtain the results in Figure 5 due to the inclusion of small aspect ratios which result in rapidly varying exponentials, and we use a 1000 term truncation of the series for the smallest values of $a$ in this case.

For the parameters given in Figure 4, the baking isotherm fails to close for Pe $\gtrsim 5.3$ (see Figure 4(c)), and this results in the melted paste region flowing through the center of the bottom boundary (see Figure $4(\mathrm{c})$ ). This means that excessively rapid lowering of the electrode results in the core region warming too slowly, with electrode paste flowing into the furnace below. Lowering the electrode more slowly allows for the paste to bake before reaching the furnace at the bottom of the domain.

The smaller the aspect ratio, the more uniform the baking, with wide cylinders more likely to develop a hole in the baking isotherm which permits electrode paste to flow into the furnace. In Figure 5, we plot the baking isotherm for multiple values of the aspect ratio, given a fixed value of the Péclet number. We then calculate the critical Péclet number, $\mathrm{Pe}^{*}$, for a given aspect ratio, such that the baking isotherm lies above $z=0$ for all $\mathrm{Pe}<\mathrm{Pe}^{*}$. To do this, we calculate $T(0,0)$ for specific $a$ and $\mathrm{Pe}$ until $T(0,0)<0$, noting the value of $\mathrm{Pe}$ for which $T(0,0) \approx 1$. We also verify that the boundary remains above the line $z=0$ (to make sure that the minimal value is indeed at $r=0$ ). We find that the baking isotherm does not close for any $a>0.74$, even as we take $\mathrm{Pe} \rightarrow 0^{+}$. 


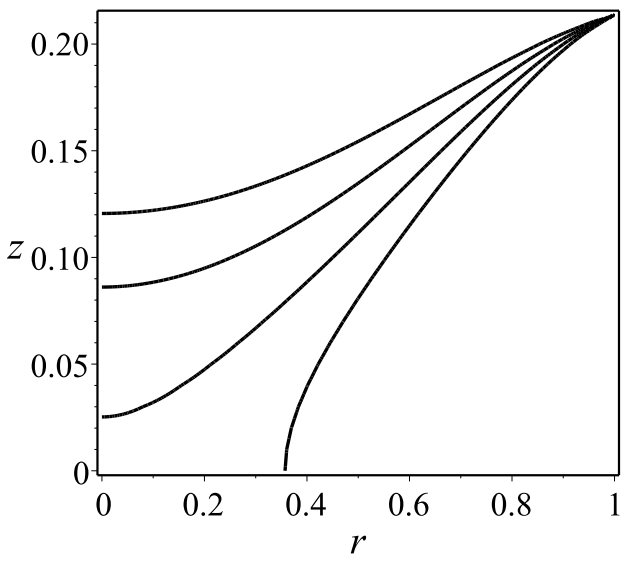

(a)

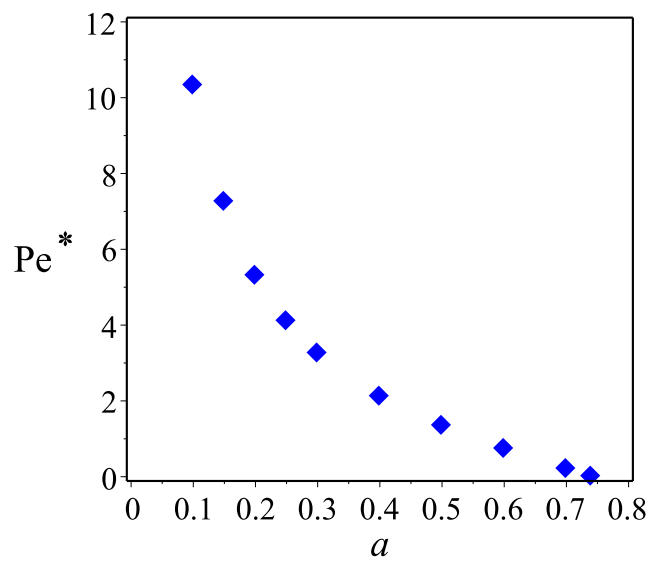

(b)

Fig. 5: (a) Plots of the baking boundaries corresponding to the $T=1$ isotherm from the solution (23) for fixed $\mathrm{Pe}=4$ for various values of the aspect ratio, $a=0.15,0.2,0.25,0.3$, with each respective isotherm decreasing in height. Note that the isotherm corresponding to $a=0.3$ fails to close. (b) Critical values $\mathrm{Pe}^{*}$ such that the baking isotherm is closed for all $\mathrm{Pe}<\mathrm{Pe}^{*}$, for a given $a$. Above this value, the baking isotherm does not close, resulting in electrode paste flowing into the furnace. Note that the critical value for $a=0.3$ is $\mathrm{Pe}^{*}=3.25$, hence why the baking isotherm corresponding to $a=0.3$ is not closed for $\mathrm{Pe}=4$ in panel (a). We fix $\mathrm{Nu}=5$ in both panels.

3.1.2. Simplified problem near the current clamps. To gain a better intuition for the current problem resulting in Joule heating, we consider a caricature of the region surrounded by the current clamps. To this end, consider a cylindrical domain $r \in[0,1], z \in\left[H_{1}, H_{2}\right]$, with current clamps along the radius and current flowing from the radial boundary downward toward a grounded bottom of the cylinder. In our caricature, we assume $T=T_{\min }<1$ at $z=H_{2}$ and $\frac{\partial T}{\partial z}=0$ at $z=H_{1}$. We approximate $T_{\text {ext }}(z)=0$ (which is a good approximation of the value $-1.754 \times 10^{-2}$ given by (13) within this region). Near $T \approx 1$, we approximate $\sigma(T) \approx \sigma(1)$. We define the parameter $\Pi=$ Po $\sigma(1)$, treating $\Pi$ as an $\mathcal{O}(1)$ parameter. We further approximate $k(T)=1$, while we take $h(z)=5$ in order to agree with (14) within the region $H_{1}<z<H_{2}$. Rescaling $z=H_{1}+\left(H_{2}-H_{1}\right) \zeta$, we note that $a /\left(H_{2}-H_{1}\right)=1$. This scales all parameters out of the current problem, which reads

$$
\begin{gathered}
\frac{1}{r} \frac{\partial}{\partial r}\left(r \frac{\partial \phi}{\partial r}\right)+\frac{\partial^{2} \phi}{\partial \zeta^{2}}=0, \\
\frac{\partial \phi}{\partial \zeta}=0 \quad \text { at } \zeta=1, \quad \phi=0 \quad \text { at } \quad \zeta=0, \quad \frac{\partial \phi}{\partial r}=1 \quad \text { at } \quad r=1, \quad \phi \text { bounded at } r=0 .
\end{gathered}
$$

Separating variables and constructing a solution in the standard manner, we find

$$
\phi(r, \zeta)=\frac{8}{\pi^{2}} \sum_{\ell=0}^{\infty} \frac{1}{(2 \ell+1)^{2}} \frac{I_{0}\left(\frac{(2 \ell+1)}{2} \pi r\right)}{I_{1}\left(\frac{(2 \ell+1)}{2} \pi\right)} \sin \left(\frac{(2 \ell+1)}{2} \pi \zeta\right)
$$

In order to gain a qualitative understanding of the Joule heating term, we calculate $|\nabla \phi|^{2}$ using a truncated 200-term series expansion from (25), plotting the result in Figure 6. The strongest Joule heating is located very close to the boundary, and is centred near $\zeta=0$ and $r=0$ in the scaled coordinates. While (25) results from a number of simplifications, the qualitative finding that Joule heating is highly localized near the current clamps, which is in qualitative agreement with the simulation shown in Figure. 3 for the full problem. 


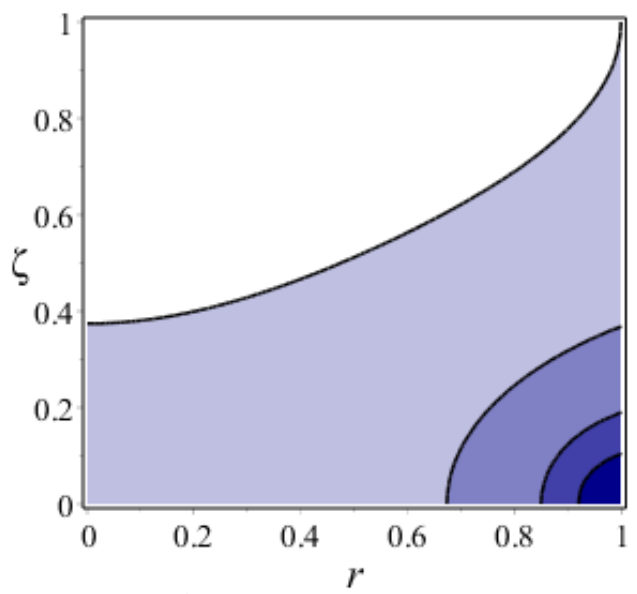

Fig. 6: Plot of the Joule heating term $|\nabla \phi|^{2}$ calculated using a 200-term series expansion (25). Contours are taken at $1,3,5,7$, respectively, with darker shading denoting stronger Joule heating. Note that the strongest Joule heating is adjacent to the boundary, beneath the current clamps, although Joule heating extends across the radius of the domain. Recall that $\zeta \in[0,1]$ corresponds to the region between the current clamps, rather than the entire electrode.

One may use (25) to compute $|\nabla \phi|^{2}$ in the relevant problem for temperature, which is

$$
-\mathrm{Pe} \frac{\partial T}{\partial \zeta}=\frac{1}{r} \frac{\partial}{\partial r}\left(r \frac{\partial T}{\partial r}\right)+\frac{\partial^{2} T}{\partial \zeta^{2}}+\Pi\left(\left(\frac{\partial \phi}{\partial r}\right)^{2}+\left(\frac{\partial \phi}{\partial \zeta}\right)^{2}\right),
$$

(26b) $T=T_{\min }$ at $\zeta=1, \quad \frac{\partial T}{\partial \zeta}=0$ at $\zeta=0, \quad T$ bounded at $r=0, \quad \frac{\partial T}{\partial r}=-5 \mathrm{Nu} T$ at $r=1$.

Writing $T_{\text {hom }}(r, \zeta)=\mathcal{R}(r) \mathcal{Z}(\zeta)$, the relevant spectral problem is given by

$$
\frac{1}{r} \frac{d}{d r}\left(r \frac{d \mathcal{R}}{d r}\right)=\gamma \mathcal{R}, \quad \mathcal{R} \text { bounded at } r=0, \quad \frac{\partial \mathcal{R}}{\partial r}=-5 \mathrm{Nu} \mathcal{R} \text { at } r=1 .
$$

The solution of problem (27) results in a Dini series, which is a type of Fourier-Bessel series associated with Robin boundary conditions. For further details, see Chapter 18 of the classical reference [48]. Note that there is no non-trivial solution for $\gamma \geq 0$, so we consider $\gamma=-\Gamma^{2}$, finding that $\mathcal{R}_{\ell}(r)=J_{0}\left(\Gamma_{\ell} r\right), \ell=1,2, \ldots$, where $0<\Gamma_{1}<\Gamma_{2}<\cdots \rightarrow \infty$ are solutions of the transcendental equation $\Gamma J_{1}(\Gamma)-5 \mathrm{Nu} J_{0}(\Gamma)=0$. By symmetry we need only consider non-negative values of $\Gamma$, and we note that $\Gamma=0$ is never a solution. For the reference value of $\mathrm{Nu}=5$, the first several eigenvalues are $\Gamma_{1}=2.310798161, \Gamma_{2}=5.306797248, \Gamma_{3}=8.326170092$, $\Gamma_{4}=11.35750185, \Gamma_{5}=14.39961798$. The corresponding solution to the problem $\mathcal{Z}_{\ell}^{\prime \prime}+\operatorname{Pe} \mathcal{Z}_{\ell}^{\prime}=\Gamma_{\ell}^{2} \mathcal{Z}_{\ell}$, $\mathcal{Z}_{\ell}^{\prime}(0)=0$ reads

$$
\mathcal{Z}_{\ell}(\zeta)=\left\{\cosh \left(\sqrt{\mathrm{Pe}^{2}+4 \Gamma_{\ell}^{2}} \frac{\zeta}{2}\right)+\frac{\mathrm{Pe}}{\sqrt{\mathrm{Pe}^{2}+4 \Gamma_{\ell}^{2}}} \sinh \left(\sqrt{\mathrm{Pe}^{2}+4 \Gamma_{\ell}^{2}} \frac{\zeta}{2}\right)\right\} \exp \left(-\frac{\mathrm{Pe}}{2} \zeta\right)
$$

We can now write $T_{\text {hom }}(r, \zeta)=\sum_{\ell=1}^{\infty} \mathcal{B}_{\ell} J_{0}\left(\Gamma_{\ell} r\right) \mathcal{Z}_{\ell}(\zeta)$, where the $\mathcal{B}_{\ell}$ are coefficients to be determined through the final condition $T=T_{\min }$ at $\zeta=1$. In order to find the $\mathcal{B}_{\ell}$ 's, we observe that at $\zeta=1$ the series is a function of $r$ alone, which must be constant. Matching coefficients of $\sum_{\ell=1}^{\infty} \mathcal{B}_{\ell} J_{0}\left(\Gamma_{\ell} r\right) \mathcal{Z}_{\ell}(1)$ with the Dini series for the constant $T_{\min }$ in term of the basis $J_{0}\left(\Gamma_{\ell} r\right)$, we finally obtain

$$
T_{\mathrm{hom}}(r, \zeta)=T_{\min } \sum_{\ell=1}^{\infty} \frac{2 J_{1}\left(\Gamma_{\ell}\right) J_{0}\left(\Gamma_{\ell} r\right)}{\Gamma_{\ell}\left(J_{0}\left(\Gamma_{\ell}\right)^{2}+J_{1}\left(\Gamma_{\ell}\right)^{2}\right)} \frac{\mathcal{Z}_{\ell}(\zeta)}{\mathcal{Z}_{\ell}(1)}
$$


The problem for $T_{\text {Joule }}$ will involve a radial basis consisting of $\left\{J_{0}\left(\Gamma_{\ell} r\right)\right\}_{\ell=1}^{\infty}$, and hence the spectrum will remain the same. The forcing function $|\nabla \phi|^{2}$ may be calculated from (25), and changing the basis of this function, we write

$$
|\nabla \phi|^{2}=\sum_{\ell=1}^{\infty} J_{0}\left(\Gamma_{\ell} r\right) \Phi_{\ell}(\zeta), \quad \Phi_{\ell}(\zeta)=\frac{2 \int_{0}^{1} r J_{0}\left(\Gamma_{\ell} r\right)|\nabla \phi|^{2} d r}{\Gamma_{\ell}\left(J_{0}\left(\Gamma_{\ell}\right)^{2}+J_{1}\left(\Gamma_{\ell}\right)^{2}\right)}
$$

Separating variables like $T_{\text {Joule }}(r, \zeta)=\mathcal{R}_{\ell}(r) \mathcal{Y}_{\ell}(\zeta)$ with $\mathcal{R}_{\ell}(r)=J_{0}\left(\Gamma_{\ell} r\right)$ for $\ell=1,2, \ldots$, we obtain the boundary value problems

$$
\frac{d^{2} \mathcal{Y}_{\ell}}{d \zeta^{2}}+\operatorname{Pe} \frac{d \mathcal{Y}_{\ell}}{d \zeta}-\Gamma_{\ell}^{2} \mathcal{Y}_{\ell}+\Pi \Phi_{\ell}(\zeta)=0, \quad \mathcal{Y}_{\ell}=0 \quad \text { at } \quad \zeta=1, \quad \frac{d \mathcal{Y}_{\ell}}{d \zeta}=0 \quad \text { at } \quad \zeta=0, \quad \ell=1,2, \ldots
$$

Employing variation of parameters, we find that solutions to (31) take the form

$$
\mathcal{Y}_{\ell}(\zeta)=\frac{\Pi}{\mathcal{Z}_{\ell}(1)}\left\{\mathcal{Z}_{\ell}(\zeta) \int_{0}^{1} \mathcal{K}_{\ell}(1, \tau) \Phi_{\ell}(\tau) d \tau-\mathcal{Z}_{\ell}(1) \int_{0}^{\zeta} \mathcal{K}_{\ell}(\zeta, \tau) \Phi_{\ell}(\tau) d \tau\right\}, \quad \ell=1,2 \ldots,
$$

where the kernels are defined by

$$
\mathcal{K}_{\ell}(\zeta, \tau)=\frac{2}{\sqrt{\mathrm{Pe}^{2}+4 \Gamma_{\ell}^{2}}} \sinh \left(\frac{1}{2} \sqrt{\mathrm{Pe}^{2}+4 \Gamma_{\ell}^{2}}(\zeta-\tau)\right) \exp \left(-\frac{\mathrm{Pe}}{2}(\zeta-\tau)\right), \quad \ell=1,2, \ldots,
$$

Summing $T_{\text {hom }}$ and $T_{\text {Joule }}$, we obtain the exact solution

$$
\begin{aligned}
T(r, \zeta)= & T_{\min } \sum_{\ell=1}^{\infty} \frac{2 J_{1}\left(\Gamma_{\ell}\right) J_{0}\left(\Gamma_{\ell} r\right)}{\Gamma_{\ell}\left(J_{0}\left(\Gamma_{\ell}\right)^{2}+J_{1}\left(\Gamma_{\ell}\right)^{2}\right)} \frac{\mathcal{Z}_{\ell}(\zeta)}{\mathcal{Z}_{\ell}(1)} \\
& +\Pi \sum_{\ell=1}^{\infty} \frac{J_{0}\left(\Gamma_{\ell} r\right)}{\mathcal{Z}_{\ell}(1)}\left(\mathcal{Z}_{\ell}(\zeta) \int_{0}^{1} \mathcal{K}_{\ell}(1, \tau) \Phi_{\ell}(\tau) d \tau-\mathcal{Z}_{\ell}(1) \int_{0}^{\zeta} \mathcal{K}_{\ell}(\zeta, \tau) \Phi_{\ell}(\tau) d \tau\right) .
\end{aligned}
$$

The function multiplying the Joule heating parameter $\Pi$ is non-negative, and takes the greatest value below the baking isotherm and near the boundary of the domain. So, the baking isotherm will tend to increase in height with Joule heating, with this increase correlated to a strengthening of the Joule heating parameter П. Later simulations will better demonstrate the quantitative strength of this effect.

3.2. Effective steady thermal problem. We turn our attention to the case where the electrode slip is periodic in time. For slips of excessive duration or velocity, the baking isotherm can fall below $z=0$, which is undesirable. Therefore, in practice a succession of slips of short duration are employed to lower the electrode. In such a case, the system starts at a steady state corresponding to no slip $(f=0)$, there is a brief slip or combination of slips which lower the baking isotherm, and then the system evolves back to the steady state corresponding to no slip $(f=0)$, with this process repeating in a periodic manner. In this configuration, we assume that $f(t)$ is periodic with period $\tau$, and that $\int_{0}^{\tau}|f(t)| d t \ll 1$. We assume that a solution to (15a) takes the form

$$
T(r, z, t)=T_{\text {mean }}(r, z)+T_{\text {var }}(r, z, t),
$$

where $T_{\text {mean }}(r, z)$ is a mean value of the temperature field over time, while $T_{\mathrm{var}}(r, z, t)$ accounts for the variations around this mean, so that $\frac{1}{\tau} \int_{t}^{t+\tau} T d t=T_{\text {mean }}$ and $\int_{t}^{t+\tau} T_{\text {var }} d t=0$. If such a mean value does not exist, then the baking isotherm will transiently drift down or up over each period $\tau$, which is undesirable. We shall assume $T_{\operatorname{var}}(r, z, t)$ and its derivatives are small relative to $T_{\text {mean }}(r, z)$. Using (35) in (15a), we have

$$
\operatorname{Pe} \frac{\partial T_{\text {var }}}{\partial t}-a \operatorname{Pe} f(t) \frac{\partial}{\partial z}\left(T_{\text {mean }}+T_{\text {var }}\right)=\nabla \cdot\left(k\left(T_{\text {mean }}\right) \nabla T_{\text {mean }}\right)+\operatorname{Po} \sigma\left(T_{\text {mean }}\right)|\nabla \phi|^{2}+\mathcal{O}\left(T_{\text {var }}\right),
$$

where the $\mathcal{O}\left(T_{\mathrm{var}}(r, z, t)\right)$ terms on the right hand side depend on $T_{\mathrm{var}}$ and its space derivatives multiplied by terms involving the time-independent $T_{\text {mean }}$ and its space derivatives. Integrating (36) over a period, and 
assuming that the terms involving $T_{\text {var }}$ are small enough to be negligible relative to corresponding terms involving the mean $T_{\text {mean }}$, at leading order we obtain an effective equation

$$
-a \mathrm{Pe}_{\mathrm{eff}} \frac{\partial T_{\text {mean }}}{\partial z}=\nabla \cdot\left(k\left(T_{\text {mean }}\right) \nabla T_{\text {mean }}\right)+\operatorname{Po} \sigma\left(T_{\text {mean }}\right)|\nabla \phi|^{2},
$$

where

$$
\mathrm{Pe}_{\mathrm{eff}}=\frac{\mathrm{Pe}}{\tau} \int_{0}^{\tau} f(t) d t
$$

is the effective Péclet number. Note that a solution $T_{\text {mean }}$ of (37) is equivalent to a solution $T$ of (18) in the case where Pe in (18) is replaced by Pe $e_{\text {eff }}$. When carrying out simulations, we compare the obtained solutions to a corresponding steady state of the stationary effective problem (37)-(38).

3.3. Simulation of time-dependent slips and comparison with effective model. Here we provide numerical simulations of the system (15), (16), (17). We choose reference parameters with guidance from our industrial partner, fixing $\mathrm{Nu}=5, a=0.14$, Po $=9.39$ (corresponding to an applied current of 130kA), corresponding to real values used for time-dependent electrode slip. The slip often occurs at a velocity $\hat{v}_{s}=3 \times 10^{-3} \mathrm{~ms}^{-1}$, so that $\mathrm{Pe}=9.72 \times 10^{2}$. While $\mathrm{Pe}$ is very large, the slip takes place over a very short time interval. We define $\Delta t$ to be the duration of a slip, and $\tau$ to be the period between when two slips are initialized. The duration of a standard slip is 5 seconds, and in dimensionless time this becomes $\Delta t=\frac{3}{1400}=2.143 \times 10^{-3}$. Then, we define $f(t)$ to be

$$
f(t)=\left\{\begin{array}{l}
1 \text { for } t \in[0, \Delta t), \\
0 \text { for } t \in[\Delta t, \tau) .
\end{array}\right.
$$

The interval between slips is on the order of an hour, which is 1.543 in dimensionless time, so the period $\tau$ is order one. We continue $f(t)$ over the positive real numbers $t>0$ by $f(t+\tau)=f(t)$; that is to say, $f(t)$ is periodic with period $\tau$. One may then modify $\tau$ so that there is a slip every hour, every half hour, and so on.

Simulations are carried out for $5 \tau$ units of time (that is, for five slips) from an initial condition computed with the stationary solver under a constant flow with $\mathrm{Pe}=\mathrm{Pe}_{\mathrm{eff}}$. Given the form of (39), we anticipated (and confirmed) that solutions were essentially periodic with maximal isotherms at $t=0, \tau, 2 \tau, \ldots, 5 \tau$, and minima offset from this by $\Delta t$, i.e., at the end of a slip.

In consultation with Elkem, we consider two cases of periodic standard slips of $5 \mathrm{~s}$ duration, as follows. Case 1: a slip occurs every 45 minutes $(\tau=1.157)$. Case 2 : a slip occurs every 25 minutes $(\tau=0.6429)$. We compare the baking and melting isotherms for the averaged cases with those of the corresponding stationary problem. For our choice of slip velocity, and using (39) in (38), we find the effective Péclet number $\mathrm{Pe}_{\mathrm{eff}}=9.72 \times 10^{2} \frac{\Delta t}{\tau}$. For each of the cases involving a standard slip, we have $\mathrm{Pe}_{\mathrm{eff}}=1.80$ (Case 1 ), $\mathrm{Pe}_{\text {eff }}=3.24$ (Case 2). We show the result of these simulations in Figure 7 by plotting the baking isotherms only (the melting isotherm behaves similarly).

We remark that over the period of a single slip, there is very little change in the baking isotherm from its maximal to minimal values. Examining a portion of these isotherms closely, we see that they fall a distance of $\mathcal{O}\left(10^{-4}\right)-\mathcal{O}\left(10^{-3}\right)$ during a slip, and return exactly (within reasonable numerical tolerance) to the effective stationary isotherm. This suggests that the problem is indeed effectively periodic, and that the effective stationary problem described in Section 3.2 is appropriate for the ranges of parameters relevant to industrial furnace operation.

In addition to making the standard slip more frequent, Elkem is interested in taking longer duration slips over a fixed time. Motivated by this, in Figure 8 we consider one slip per hour, but increase the duration of the slip from 5 seconds to 2 minutes. The industrially-relevant slip time of 5 seconds is comparable to the examples shown in Figure 7, where the effective stationary solution is effectively a maximal isotherm that is periodically returned to. The solution corresponding to a 2 minute slip is shown after a period of five slips just before a sixth, with the baking isotherm failing to close at the center of the electrode, leading to a soft breakage. We remark that the effective stationary model is a good indicator of the feasibility of an electrode set-up, as the difference between the stationary isotherms for different parameters (as shown in Section 3.4) is orders of magnitude larger than transient differences observed over the duration of a single slip. 


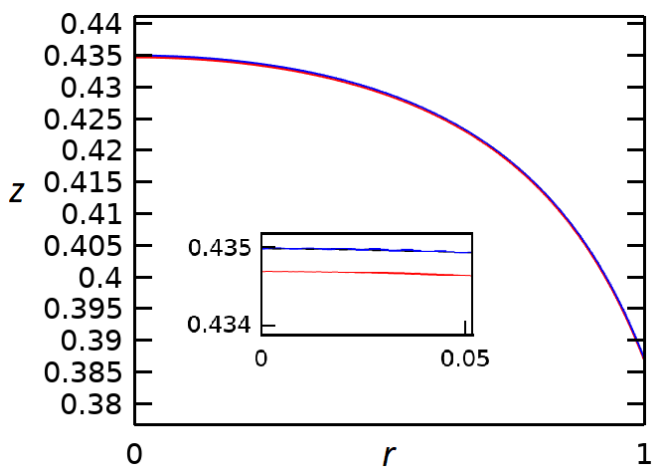

(a)

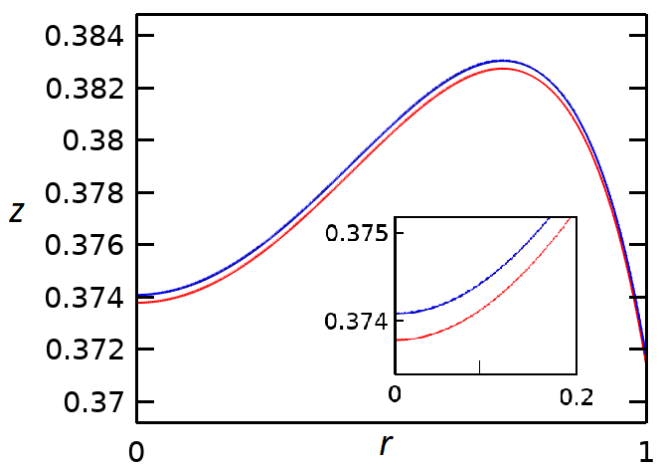

(b)

Fig. 7: Maximal (blue) and minimal (red) baking isotherms for simulations with time dependent slip of the electrode according to (39) with slip duration of 5 seconds $\left(\Delta t=2.143 \times 10^{-3}\right)$ every (a) 45 minutes $(\tau=1.157)$ and (b) 25 minutes $(\tau=0.6429)$. We take a slip rate of $\hat{v}_{s}=3 \times 10^{-3} \mathrm{~ms}^{-1}$ so that $\mathrm{Pe}=9.72 \times 10^{2}$ over the short duration of the slip. The other parameters are fixed at $\mathrm{Nu}=5, a=0.14$, $\mathrm{Po}=9.39(130 \mathrm{kA})$. We also plot the effective isotherms (black) for steady states correspond to solutions of the effective problem (37)-(38), noting that this is essentially identical to the maximal isotherm in all cases. The insets show a zoomed in view near $r=0$ that that the difference between maximal and minimal extent of the baking isotherm can be seen.

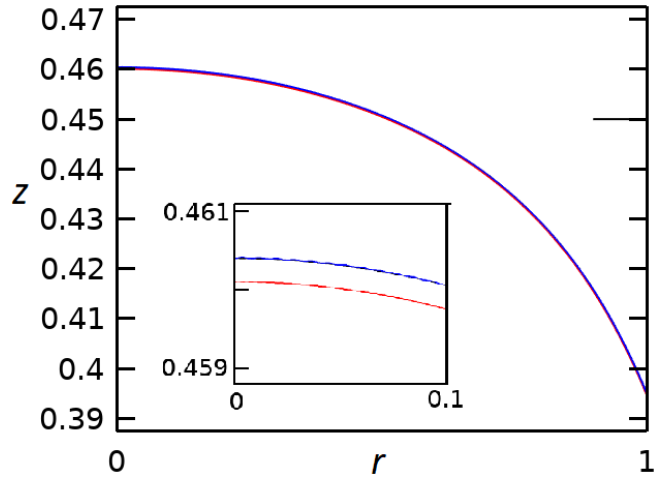

(a)

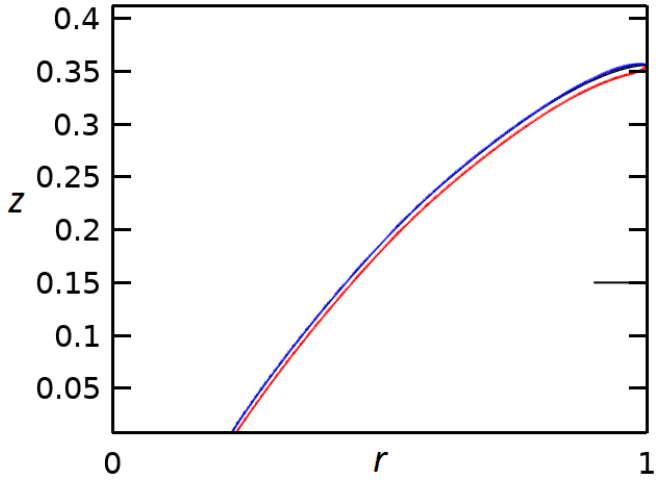

(b)

Fig. 8: Maximal (blue) and minimal (red) baking isotherms for simulations with time dependent slip of the electrode according to (39) with one slip every hour $(\tau=1.543)$ yet of varying durations of (a) 5 seconds $\left(\Delta t=2.143 \times 10^{-3}\right)$, and (b) 2 minutes $\left(\Delta t=5.143 \times 10^{-2}\right)$. We take a slip rate of $\hat{v}_{s}=3 \times 10^{-3} \mathrm{~ms}^{-1}$ so that $\mathrm{Pe}=9.72 \times 10^{2}$ over the short duration of the slip. The other parameters are fixed at $\mathrm{Nu}=5, a=0.14$, $\mathrm{Po}=9.39(130 \mathrm{kA})$. We also plot the effective isotherms (black) for steady states correspond to solutions of the effective problem (37)-(38).

3.4. Parameter sweeps for uniform slips. Having shown that time periodic slips result in solutions akin to those found for an effective uniform slip, we now carry out parameter sweeps using a uniform slip rate in order to better understand the influence of the dimensionless groups on the position of the melting and baking isotherms. We set $f(t)=1$ in (15a). Although the fluid mechanics problem is quite simple, recall that the parameter $\hat{v}_{s}$ still enters into Pe, and thus heat is still advected downward with the uniform flow, despite the simplifications.

In Figures 9-11, we plot the melting and baking isotherms for various values of the Péclet number Pe (measuring the lowering rate of the electrode), the aspect ratio $a$ (measuring the width of the electrode), and the Pomerantsev number Po (measuring the strength of the Joule heating). We find that the general qualitative trends seen in the analytical solutions of Section 3.1 for more idealized simpler cases still hold. 


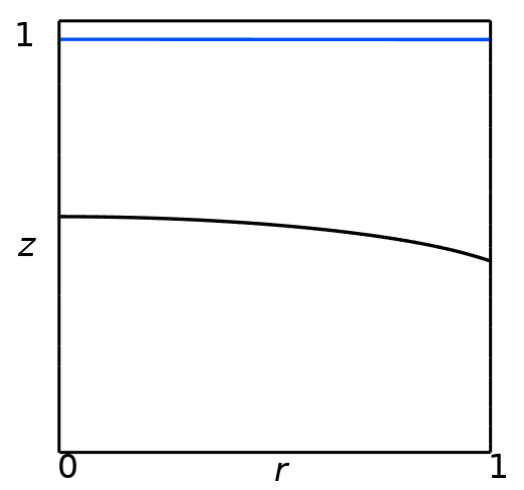

(a) $\mathrm{Pe}=0$

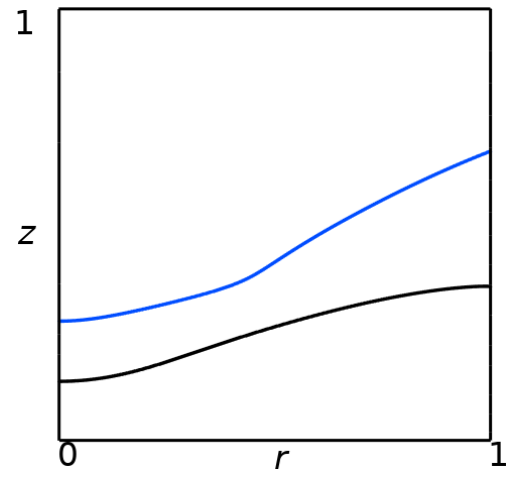

(b) $\mathrm{Pe}=20$

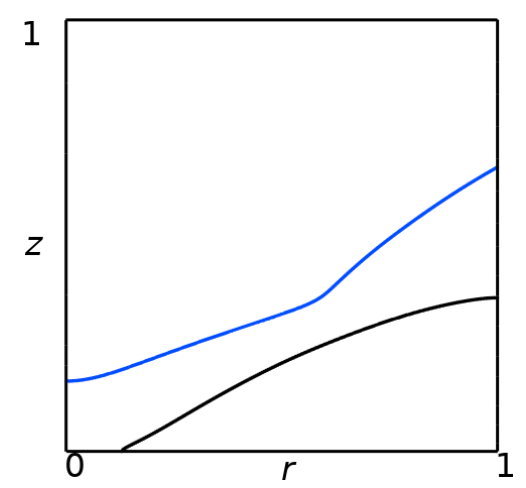

(c) $\mathrm{Pe}=35$

Fig. 9: Melting (blue) and baking (black) isotherms under a parameter sweep over the Péclet number Pe as indicated. We fix $a=0.14$ and $\mathrm{Po}=9.38$.

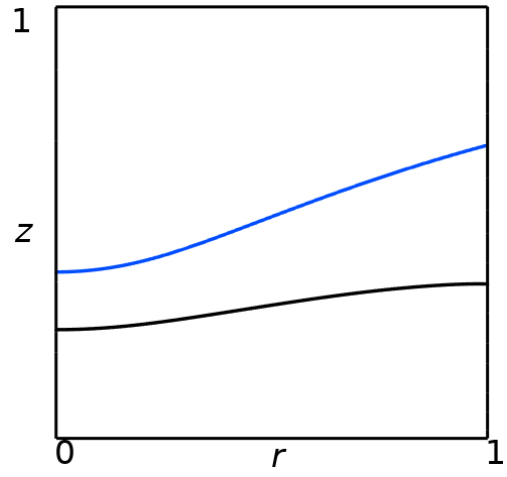

(a) $a=0.1$

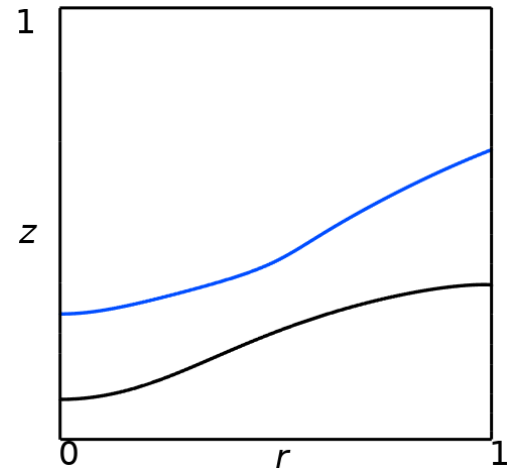

(b) $a=0.2$

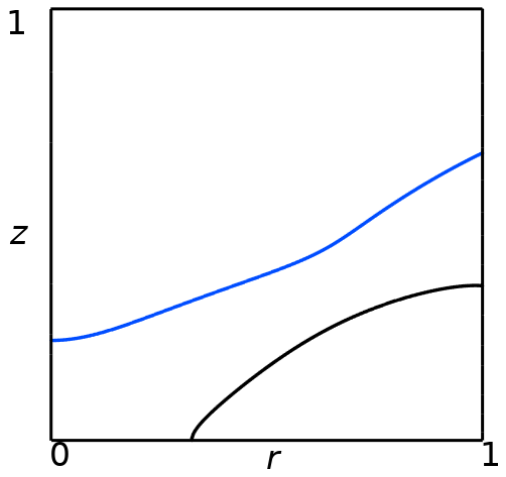

(c) $a=0.3$

Fig. 10: Melting (blue) and baking (black) isotherms under a parameter sweep over the aspect ratio $a$ as indicated. We fix $\mathrm{Pe}=15$ and $\mathrm{Po}=9.38$.

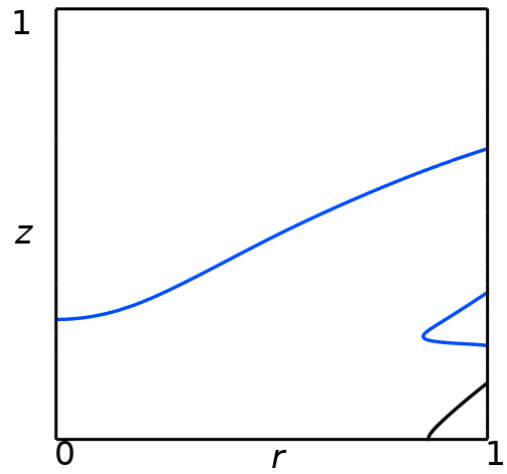

(a) $\mathrm{Po}=0$

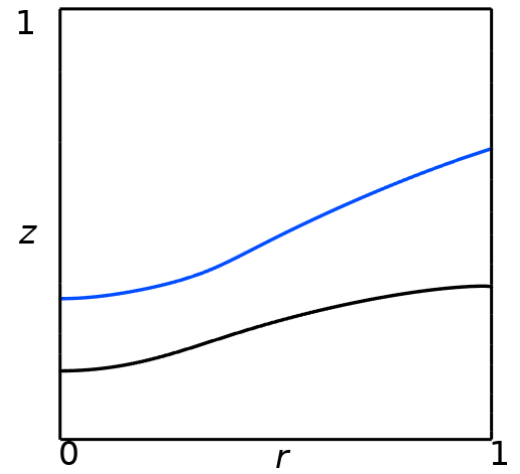

(b) $\mathrm{Po}=3.58$

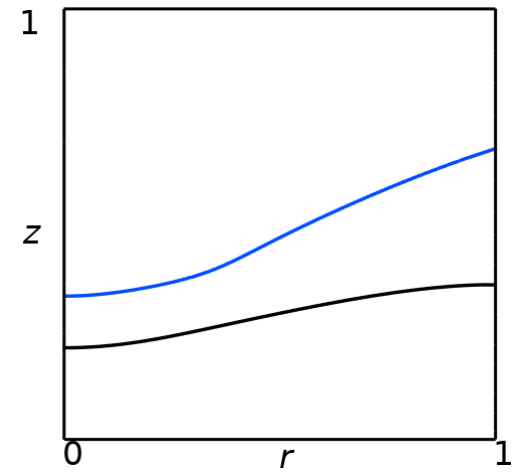

(c) $\mathrm{Po}=14.22$

Fig. 11: Melting (blue) and baking (black) isotherms under a parameter sweep over the Pomerantsev number $\mathrm{Po}$ as indicated. We fix $\mathrm{Pe}=15$ and $a=0.14$. 
As the Péclet number increases in Figure 9 (due to more rapid lowering of the electrode), the position of the baking isotherm falls lower until the point at which it does not close. Meanwhile, as the aspect ratio is increased in Figure 10 (so that the electrode becomes wider), the position of the baking isotherm lowers, since it is harder to warm progressively wider cylinders in a fixed time. Within the industrially-relevant values of the Pomerantsev number Po, there is only a small variation in either isotherm, as seen in Figure 11. However for no Joule heating $\left(\mathrm{Po}_{\mathrm{O}}=0\right)$, we note a substantial impact on the qualitative behaviour, with the baking isotherm failing to close for smaller values of $\mathrm{Pe}$, and not forming at all for larger Pe. Additionally, in the $\mathrm{Po}=0$ case, we observe the addition of a second melting isotherm near the current clamps, which forms due to the cold boundary condition near the current clamps when the current clamps are not in use. In practice, the melting region is more diffuse, and this colder region near the clamps may still behave as a fluid rather than a solid, and hence this small region does not tend to inhibit the overall flow of the electrode material.

4. Discussion and extensions. We have formulated a fairly general model for the lowering of a Søderberg electrode into a furnace, and then studied in some detail two reductions of this model: a case where the electrode is lowered continuously yet slowly, and a second case were the electrode is lowered periodically, with the motion being more rapid during the lowering. For reasonable parameter values corresponding to realistic furnace operation, we find that the effective theory derived from the periodically lowered case gives results which are in good agreement with the continuously lowered electrode model. Hence, the process of lowering a Søderberg electrode is adequately modelled as a steady-state process, provided that the baking isotherm closes.

We can draw several conclusions regarding optimal furnace operation from our analytical solutions and numerical simulations. There is an obvious desire to utilize the maximal amount of material in a given time. However, if the electrode is lowered too fast then liquid paste may seep into the furnace, leading to soft breakage which results in costly damage. To mitigate this, the operator should choose to lower the electrode at a rate which permits the baking isotherm to close properly, and hence the electrode to fully bake, before the paste reaches the bottom of the domain, which corresponds to the point where electrode material enters the furnace. Our analytical and numerical results suggest that there is a range of permissible Péclet numbers, $\mathrm{Pe} \in\left[0, \mathrm{Pe}^{*}\right)$, with $\mathrm{Pe}^{*}$ the critical value at which the baking isotherm barely closes right at the bottom $(z=0)$ of the domain. The position of the baking isotherm appears to be monotone in the Péclet number (fixing all other parameters, of course), and hence so long as the value is below the critical value, the furnace will continue to operate normally. If the Péclet number exceeds this value, then costly damage may result. The electrode may therefore be lowered up to some critical effective velocity, before the process fails. Our findings highlight the key role that the Péclet number plays in realistic thermistor problems, and complements the expansive literature on static thermistor problems for which $\mathrm{Pe}=0$.

Similarly, the aspect ratio of the cylindrical electrode will influence where the baking isotherm lies. If the aspect ratio is small enough, boundary heating due to both the applied current and regions of warm external temperature, will result in heat flow through to the center of the cylinder, allowing the baking isotherm to close. On the other hand, if the cylinder is too wide, then these transport mechanisms may not adequately heat the electrode paste. This is an important consideration if the development of wider configurations is considered. Combining the parameter dependences, our results suggest that wider electrodes will be viable only if they are lowered more slowly into the furnace. Due to this reduction in rate of lowering, it is not clear if a wider electrode would provide more or less material per unit time to the furnace below, and this likely merits further study.

The Pomerantsev number, Po, is a measure of the effectiveness of the Joule heating due to current applied to the boundary in a ring around the current clamps. As this dimensionless group increases, the position of the baking isotherm likewise increases (in height) in a monotone manner. In real applications, there is a practical limit to the current which may be applied at the clamps, due to both the cost of electricity and potential damage to the current clamps resulting from excessive current without sufficient heating. Therefore, although the Joule heating aids in the baking of the boundary, failure of a baking isotherm to close due to an excessively high effective slip velocity cannot always be mitigated by increasing the applied current.

There are a number of ways in which our results might be extended in future work. In addition to preventing soft breakages, there is also the possibility of hard breakages (see Figure 2), with melted paste flowing up past the solid electrode, and segregating. One way to reduce such a problem is by focusing on 
the extreme viscosity variation between the solid electrode and the melted paste, which results in a hybrid extensional and lubrication flow model coupled to a temperature model through a highly temperature dependent viscosity function. Furthermore, in flows with reacting chemicals, the local chemical concentration is known to modify the viscosity of the overall flow [33, 46], and viscosity is often modelled through exponential dependences on the particular quantities of chemicals present $[17,16]$. In some sense this is a more precise way to account for phase changes that are modelled in bulk via our non-monotone viscosity - temperature relation, and incorporating chemical reactions taking place during baking may lead to a more quantitatively accurate model.

While the assumption of an incompressible flow implied a spatially uniform velocity field for our geometry, in real electrodes they may be some degree of non-uniformity due to such density variations. That said, consideration of fully compressible Navier-Stokes equations may be excessive. As a compromise, one may consider the Boussinesq approximation, assuming density variations are small and linear. While density varies with temperature, it does so fairly gradually, with the thermal expansion $\alpha=\mathcal{O}\left(10^{-5}\right)$, with the result being only a roughly $10 \%$ change in density over the entire temperature range considered. Generally, the Boussinesq approximation is valid when $\alpha$ is small and the temperature variations are sufficiently small. For our case, not only is $\alpha$ small, but the observed density variations with pressure are linear or sub-linear (depending upon the thermal regime). The resulting buoyancy term will be most useful in the less viscous region between the melting and baking boundaries.

There are a variety of other processes of industrial importance which are similar to that we have considered here. These include flows in a slag-cleaning furnace [49], the smelting of nickel matte in a furnace [40], the electroslag remelting process [32], the production of ferronickel in electric arc furnaces [24], and electric smelting furnaces used in the platinum recovery process [6]. Given appropriate changes in the constitutive relations and parameters, the model we developed in equations (15)-(17) could be applied to such problems. We have studied part of the process of lowering a Søderberg electrode into a furnace. In the future, our model could be coupled to existing models approximating the dynamics within a furnace [44, 43], in order to more accurately model the energy transfer from the outside current clamps into the furnace via one or more Søderberg electrodes.

Acknowledgments. We thank A. Autruffe, B. M. Sloman, A. M. Valderhaug (all Elkem), C. P. Please, C. M. Rooney, and J. G. Williams (all Oxford) for helpful discussions and comments, and Elkem in particular for internal data which motivated various constitutive laws in our mathematical models. A. Kamilova was supported in part by the EPSRC Centre for Doctoral Training in Industrially Focused Mathematical Modelling (EP/L015803/1) in collaboration with Elkem.

\section{REFERENCES}

[1] S. Antontsev And M. Chipot, The thermistor problem: existence, smoothness uniqueness, blowup, SIAM Journal on Mathematical Analysis, 25 (1994), pp. 1128-1156.

[2] G. K. BAtchelor, An introduction to fluid dynamics, Cambridge University Press, 1967.

[3] T. Bergstrøm, S. Cowley, A. C. Fowler, And P. E. Seward, Segregation of carbon paste in a smelting electrode, IMA Journal of Applied Mathematics, 43 (1989), pp. 83-99.

[4] A. Bermúdez, J. Bullón, And F. Pena, A finite element method for the thermoelectrical modelling of electrodes, Communications in numerical methods in engineering, 14 (1998), pp. 581-593.

[5] J. Beukes, H. Roos, L. Shoko, P. Van Zyl, H. Neomagus, C. Strydom, and N. Dawson, The use of thermomechanical analysis to characterise söderberg electrode paste raw materials, Minerals Engineering, 46 (2013), pp. 167-176.

[6] J. J. Bezuidenhout, Computational fluid dynamic modelling of an electric smelting furnace in the platinum recovery process, PhD thesis, Stellenbosch: Stellenbosch University, 2008.

[7] C. Burstedde, O. Ghattas, G. Stadler, T. Tu, and L. C. Wilcox, Parallel scalable adjoint-based adaptive solution of variable-viscosity stokes flow problems, Computer Methods in Applied Mechanics and Engineering, 198 (2009), pp. 1691-1700.

[8] X. Chen and A. Friedman, The thermistor problem for conductivity which vanishes at large temperature, Quarterly of applied mathematics, 51 (1993), pp. 101-115.

[9] G. CimatTi, A bound for the temperature in the thermistor problem, IMA Journal of Applied Mathematics, 40 (1988), pp. $15-22$.

[10] G. CImAтtI, Remark on existence and uniqueness for the thermistor problem under mixed boundary conditions, Quarterly of Applied Mathematics, 47 (1989), pp. 117-121.

[11] A. Fitt And J. Aitchison, Determining the effective viscosity of a carbon paste used for continuous electrode smelting, Fluid dynamics research, 11 (1993), p. 37.

[12] A. FitT AND P. Howell, The manufacture of continuous smelting electrodes from carbon-paste briquettes, Journal of 
Engineering Mathematics, 33 (1998), pp. 353-376.

[13] A. Fowler, I. Frigand, AND S. Howison, Temperature surges in current-limiting circuit devices, SIAM Journal on Applied Mathematics, 52 (1992), pp. 998-1011.

[14] M. Gockenbach And K. Schmidtke, Newton's law of heating and the heat equation, Involve, a Journal of Mathematics, 2 (2009), pp. 419-437.

[15] T. Hannesson, The Si process Drawings, 2016.

[16] S. Hejazi And J. AzAiez, Stability of reactive interfaces in saturated porous media under gravity in the presence of transverse flows, Journal of Fluid Mechanics, 695 (2012), pp. 439-466.

[17] S. Hejazi, P. Trevelyan, J. Azaiez, And A. De Wit, Viscous fingering of a miscible reactive $a+b \rightarrow c$ interface: $a$ linear stability analysis, Journal of Fluid Mechanics, 652 (2010), pp. 501-528.

[18] S. Howison, A note on the thermistor problem in two space dimensions, Quarterly of Applied Mathematics, 47 (1989), pp. 509-512.

[19] S. Howison, J. Rodrigues, And M. Shillor, Stationary solutions to the thermistor problem, Journal of Mathematical Analysis and Applications, 174 (1993), pp. 573-588.

[20] R. Innvaer, The Søderberg electrode system. Recent research and development. New challenges., in International FerroAlloys Congress, New Orleans, 1989, pp. 216-226.

[21] R. Innver, K. Fidje, And T. SirA, 3-dimensional calculations on smelting electrodes, Modeling, Identification and Control, 8 (1987), pp. 103-115.

[22] R. InNvaer, K. Fidje, AND R. UGLand, Effect of current variations on material properties and thermal stresses in söderberg electrodes, Proc. of INFACON IV. Rio de Janeiro, Brasil, (1986), pp. 321-330.

[23] R. Innver, A. VAtland, And L. Olsen, Operational parameters for soderberg electrodes from calculations, measurements, and plant experience, in Mintek 50, Johannesburg 1984, 1985.

[24] K. Karalis, N. Karkalos, G. Antipas, and A. Xenidis, Pragmatic analysis of the electric submerged arc furnace continuum, Royal Society Open Science, 4 (2017), p. 170313.

[25] A. LACEY, Thermal runaway in a non-local problem modelling ohmic heating: Part $i$ : Model derivation and some special cases, European Journal of Applied Mathematics, 6 (1995), pp. 127-144.

[26] A. LACEY, Thermal runaway in a non-local problem modelling ohmic heating. part ii: General proof of blow-up and asymptotics of runaway, European Journal of Applied Mathematics, 6 (1995), pp. 201-224.

[27] A. LACEY, Thermo-electrical stability in an electrode, Maths in Industry, (1998).

[28] L. Landau and E. Lifshitz, Course of theoretical physics. vol. 6: Fluid mechanics, Pergamon Press, 1959.

[29] B. Larsen, J. P. M. Amaro, S. Z. Nascimento, K. Fidje, and H. Gran, Melting and densification of electrode paste briquettes in Søderberg electrodes, Elkem, (1998), pp. 1-10.

[30] B. Larsen, H. Feldborg, and S. Halvorsen, Minimizing thermal stress during shutdown of Soderberg electrodes, in The thirteenth International Ferroalloys Congress Efficient technologies in ferroalloy industry, Almaty, Kazakhstan, 2013, pp. 453-467.

[31] H. LARSEn, Current distribution in the electrodes of industrial three-phase electric smelting furnaces, in Proceedings of the 2006 Nordic COMSOL Conference, 2006.

[32] B. Li, B. WANG, AND F. Tsukinashi, Modeling of electromagnetic field and liquid metal pool shape in an electroslag remelting process with two series-connected electrodes, Metallurgical and Materials Transactions B, 45 (2014), pp. 1122-1132.

[33] O. Manickam And G. Homsy, Simulation of viscous fingering in miscible displacements with nonmonotonic viscosity profiles, Physics of Fluids, 6 (1994), pp. 95-107.

[34] I. Mc Dougall, C. Smith, B. Olmstead, and W. Gericke, A finite element model of a søderberg electrode with an application in casing design, Proc. of INFACON X. Cape Town, South Africa, (2004), pp. 575-583.

[35] R. Meyjes, J. Venter, and U. Van Rooyen, Advanced modelling and baking of electrodes, in The Twelfth International Ferroalloys Congress: Sustainable Future, Helsinki, 2010, pp. 779-788.

[36] L. R. Nelson And F. X. PRIns, Insights into the influences of paste additions and levels on Søderberg electrode management, in Tenth International Ferroalloys Congress, vol. 1, Cape Town, 2004, pp. 418-431.

[37] H. Plsson And M. R. Jnsson, Finite element analysis of proximity effects in Sderberg electrodes, tech. report, 2000.

[38] C. R. RAY, P. K. SAhoo, And S. S. RAO, Electrode management-investigation into soft breaks at $48 \mathrm{MVa} \mathrm{FeCr}$ closed furnace, in Innovations in Ferro Alloy Industry, 2007, pp. 740-751.

[39] L. Roberts, E. Nordgård-Hansen, Ø. Mikkelsen, S. A. Halvorsen, and R. A. Van Gorder, A heat and mass transfer study of carbon paste baking, International Communications in Heat and Mass Transfer, 88 (2017), pp. 9-19.

[40] Y. Sheng, G. Irons, And D. Tisdale, Transport phenomena in electric smelting of nickel matte: Part ii. mathematical modeling, Metallurgical and Materials Transactions B, 29 (1998), pp. 85-94.

[41] L. Shoko, J. Beukes, and C. Strydom, Determining the baking isotherm temperature of söderberg electrodes and associated structural changes, Minerals Engineering, 49 (2013), pp. 33-39.

[42] A. Skjeldestad, M. Tangstad, L. Lindstad, and B. Larsen, Temperature profiles in søderberg electrodes, in Production Technologies and Operation, Kiev, 2015. The Fourteenth International Ferroalloys Congress, 2015, pp. 327-338.

[43] B. M. Sloman, C. P. Please, and R. A. Van Gorder, Asymptotic analysis of a silicon furnace model, SIAM Journal on Applied Mathematics, 78 (2018), pp. 1174-1205.

[44] B. M. Sloman, C. P. Please, R. A. Van Gorder, A. M. Valderhaug, R. G. Birkeland, and H. Wegge, A heat and mass transfer model of a silicon pilot furnace, Metallurgical and Materials Transactions B, 48 (2017), pp. $2664-2676$.

[45] C. W. SöDerberg, Electrode for electric furnaces and process for manufacturing the same, 1923, https://patentimages. storage.googleapis.com/87/af/9c/f05dace9cc857a/US1440724.pdf.

[46] C. Tan and G. Homsy, Stability of miscible displacements in porous media: Rectilinear flow, The Physics of Fluids, 29 (1986), pp. 3549-3556.

[47] K. TORKLEP, Viscometry in paste production, tech. report, 1988.

[48] G. N. Watson, A treatise on the theory of Bessel functions, Cambridge University Press, 1995. 
[49] J. Xia and T. Ahokainen, Numerical modelling of slag flows in an electric furnace, Scandinavian Journal of Metallurgy, 33 (2004), pp. 220-228. 